\title{
A Facile Domino Access to Chiral Bicyclo[3.2.1]octanes and Discovery of a New Catalytic Activation Mode
}

\author{
Bin Tan, Yunpeng Lu, Xiaofei Zeng, Pei Juan Chua, and Guofu Zhong* \\ Division of Chemistry and Biological Chemistry, School of Physical \& Mathematical Sciences, Nanyang Technological \\ University, 21 Nanyang Link, Singapore 637371, Singapore
}

\section{Supporting Information}

X-ray crystallographic determination of $\mathbf{3 f}$

Table. Electronic and free energies of the transition states for the Michael addition catalyzed by

catalyst VI by the DFT calculations

Figure. Located transition state structures with distant parameters in angstroms using quinine amine

catalyst VI (most H atoms are removed for clarity)

S4

General Information

S5

Typical Procedure for Michael-Henry Reactions

S6

Characterization of the Michael-Henry Products

S6

General procedure for the preparation of (1a) and (1b)

S17

DFT calculation

References

S90 

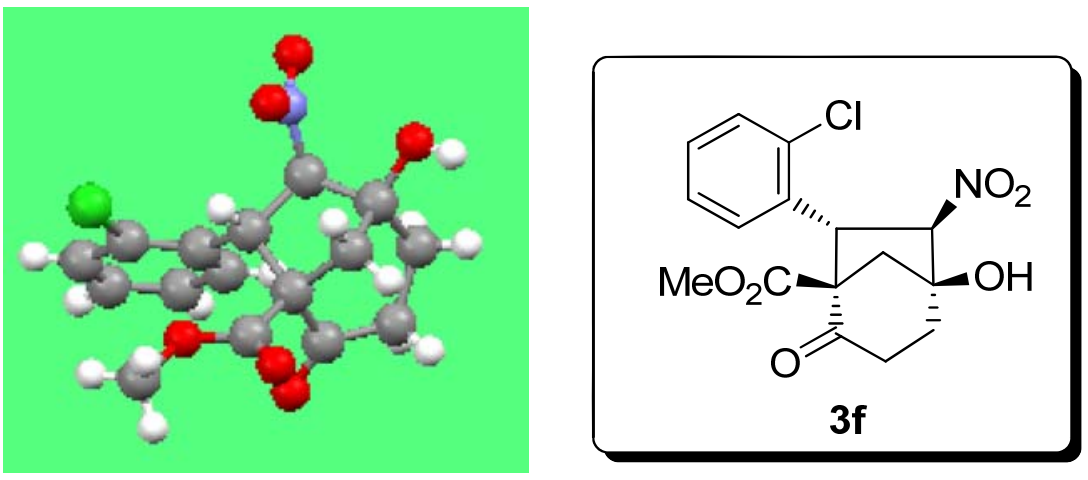

X-ray crystallographic determination of 3f (\#CCDC 719547). 


\section{Theoretical Study of the Transition State Structures and Mechanism Considerations}

Geometry optimizations were performed using the B3LYP-DFT procedure. ${ }^{1}$ As for the basis set, $6-31 G(d)$ basis $^{2}$ are used for all atoms except the protons in the amine groups, which uses 6-31G(d,p) basis. ${ }^{3}$ All transition state structures have been validated by vibrational analysis with the same level of theory/basis and have one imaginary vibrational frequency. All calculations have been done with Gaussian $03{ }^{4}$

\section{(The following Figures are the Transition States using DFT calculation)}

Table. Electronic and free energies of the transition states for the Michael addition catalyzed by catalyst VI by the DFT calculations

\begin{tabular}{ccccc}
\hline $\begin{array}{c}\text { Transiton } \\
\text { state }\end{array}$ & $\begin{array}{c}\text { Eelec } \\
\text { (Hartree) }\end{array}$ & $\begin{array}{c}\Delta \text { Eelec } \\
(\mathrm{kcal} / \mathrm{mol})\end{array}$ & $\begin{array}{c}\mathrm{G} \\
\text { (Hartree) }\end{array}$ & $\begin{array}{c}\Delta \mathrm{G} \\
\text { (Hartree) }\end{array}$ \\
\hline TS-1 $(S, S)$ & -3539.2775 & 8.53 & -3538.5237 & 7.22 \\
TS-2 $(S, R)$ & -3539.2759 & 9.54 & -3538.5181 & 10.7 \\
TS-3 $(R, S)$ & -3539.2742 & 10.6 & -3538.5174 & 11.2 \\
TS-4 $(R, R)$ & -3539.2699 & 13.3 & -3538.5130 & 13.9 \\
TS-5 (R,R) & -3539.2771 & 8.79 & -3538.5225 & 7.97 \\
TS-6 (R,S) & -3539.2911 & 0.00 & -3538.5352 & 0.00 \\
TS-7 $(S, R)$ & -3539.2820 & 5.71 & -3538.5265 & 5.44 \\
TS-8 $(S, S)$ & -3539.2717 & 12.7 & -3538.5169 & 11.5 \\
\hline
\end{tabular}



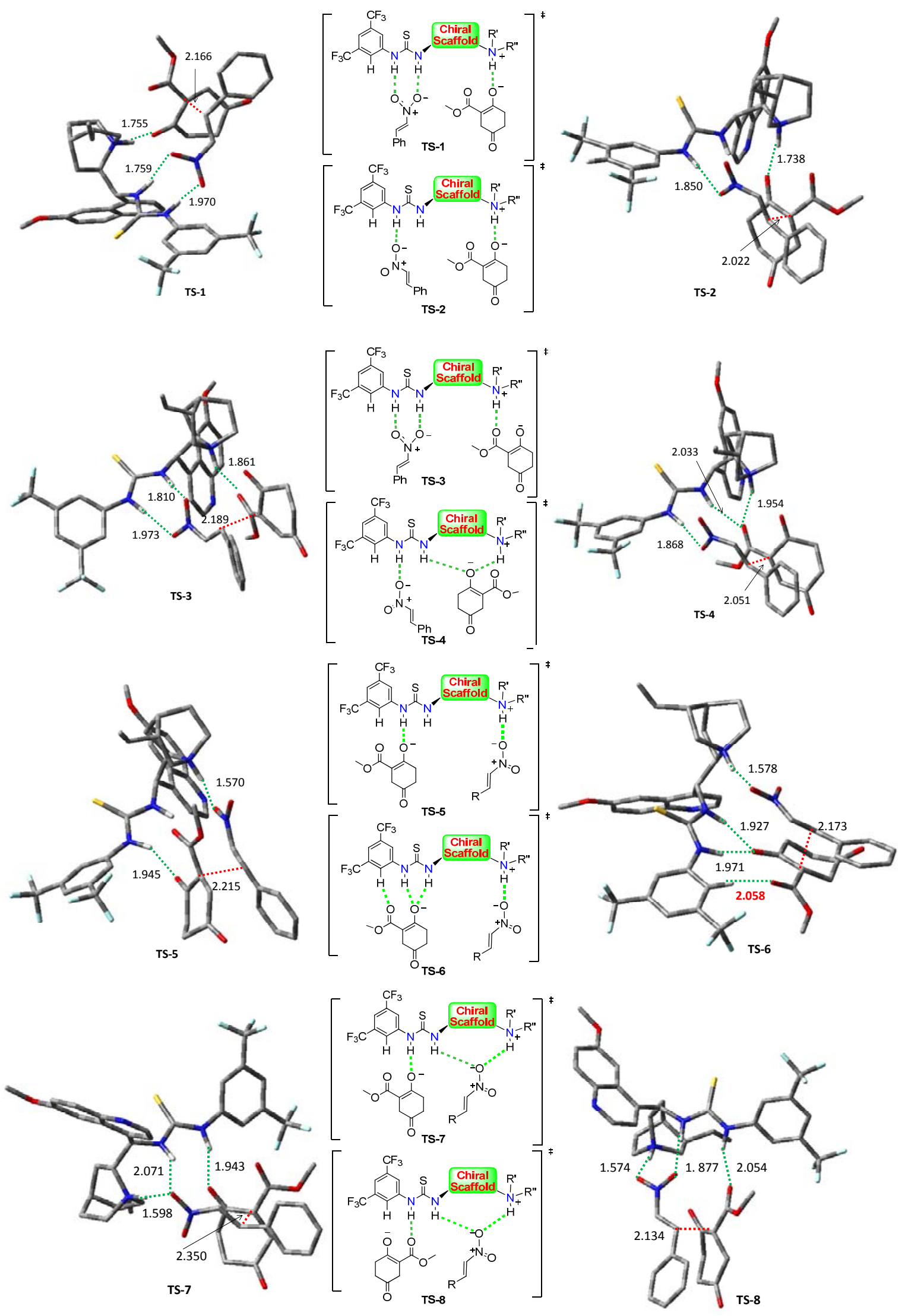

Figure. Located transition state structures with distant parameters in angstroms using quinine amine catalyst VI (most H atoms are removed for clarity). 
General Information: Analytical thin layer chromatography (TLC) was performed using Merck 60 F254 precoated silica gel plate $(0.2 \mathrm{~mm}$ thickness $)$. Subsequent to elution, plates were visualized using UV radiation (254 nm) on Spectroline Model ENF-24061/F $254 \mathrm{~nm}$. Further visualization was possible by staining with basic solution of potassium permanganate or acidic solution of ceric molybdate.

Flash chromatography was performed using Merck silica gel 60 with freshly distilled solvents. Columns were typically packed as slurry and equilibrated with the appropriate solvent system prior to use.

Proton nuclear magnetic resonance spectra $\left({ }^{1} \mathrm{H}\right.$ NMR) were recorded on Bruker AMX 400 spectrophotometer $\left(\mathrm{CDCl}_{3}\right.$ as solvent). Chemical shifts for ${ }^{1} \mathrm{H}$ NMR spectra are reported as $\delta$ in units of parts per million (ppm) downfield from $\mathrm{SiMe}_{4}(\delta 0.0)$ and relative to the signal of chloroform-d ( $\delta 7.26$, singlet). Multiplicities were given as: $\mathrm{s}$ (singlet), $\mathrm{d}$ (doublet), $\mathrm{t}$ (triplet), dd (double of doublet) or $m$ (multiplets). The number of protons (n) for a given resonance is indicated by $\mathrm{nH}$. Coupling constants are reported as a $J$ value in Hz. Carbon nuclear magnetic resonance spectra $\left({ }^{13} \mathrm{C} N \mathrm{NM}\right)$ are reported as $\delta$ in units of parts per million $(\mathrm{ppm})$ downfield from $\mathrm{SiMe}_{4}(\delta 0.0)$ and relative to the signal of chloroform-d $(\delta$ 77.0, triplet).

Enantioselectivities were determined by High performance liquid chromatography (HPLC) analysis employing a Daicel Chiralpak AS-H column. Optical rotations were measured in $\mathrm{CH}_{2} \mathrm{Cl}_{2}$ on a Schmidt + Haensdch polarimeter (Polartronic MH8) with a $1 \mathrm{~mL}$ cell (c given in $\mathrm{g} / 100 \mathrm{~mL}$ ). Absolute configuration of the products was determined by X-ray.

High resolution mass spectrometry $($ HRMS) was recorded on Finnigan MAT $95 \times \mathrm{P}$ spectrometer. 
Racemates were synthesized using Quinine and Quinidine thiourea derivatives (1:1 ratio)

\section{Typical Procedure for Michael-Henry Reactions:}

Catalyst VI $(0.005$ mmol, 0.05 eq $)$ was added to a solution of methyl 2,5-dioxocyclohexanecarboxylate 1a $(0.2 \mathrm{mmol}, 2 \mathrm{eq})$ and nitroolefins $(0.1 \mathrm{mmol}, 1 \mathrm{eq})$ in benzonitrile $(0.5 \mathrm{~mL})$ at room temperature. The resulting mixture was stirred vigorously. After the reaction was complete (monitored by TLC and crude NMR), the products were afforded by flash chromatography over silica gel (EtOAc : Hexane $=1: 4$ to $1: 2$ ).

\section{Characterization of the Michael-Henry Products}

$(1 R, 5 S, 6 R, 7 S)$-methyl 5-hydroxy-6-nitro-2-oxo-7-phenylbicyclo[3.2.1]

octane-1-carboxylate (3a)

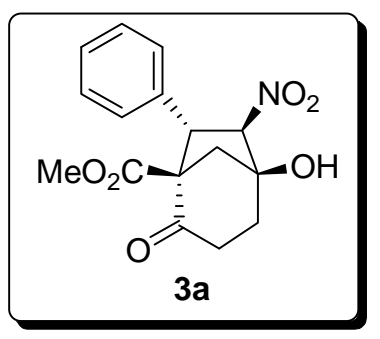

The title compound was prepared according to the typical procedure, as described above in $93 \%$ yield.

${ }^{1} \mathrm{H}-\mathrm{NMR}\left(400 \mathrm{MHz}, \mathrm{CDCl}_{3}\right) \delta$ 7.36-7.29 (m, 3H), $7.21(\mathrm{~d}, J=7.6 \mathrm{~Hz}, 2 \mathrm{H}), 5.27(\mathrm{~d}, J=5.6$ $\mathrm{Hz}, 1 \mathrm{H}), 4.88(\mathrm{~d}, J=5.6 \mathrm{~Hz}, 1 \mathrm{H}), 3.86(\mathrm{~s}, 3 \mathrm{H}), 3.01(\mathrm{dd}, J=3.6,12.8 \mathrm{~Hz}, 1 \mathrm{H}), 2.64(\mathrm{brs}$, 1H), 2.61-2.53 (m, 2H), 2.30-2.17 (m, 2H), 2.13-2.04 (m, 1H).

${ }^{13} \mathrm{C}-\mathrm{NMR}\left(100 \mathrm{MHz}, \mathrm{CDCl}_{3}\right) \delta 203.4,169.7,135.7,129.0,127.8,127.1,98.6,76.3,64.9$, $53.0,52.3,44.8,37.0,35.2$.

HPLC: Chiralpak AD-H (hexane $/ i-\mathrm{PrOH}=85 / 15$, flow rate $1 \mathrm{~mL} / \mathrm{min}, \lambda=210 \mathrm{~nm}$ ), $t_{\mathrm{R}}$ 
$($ major $)=9.0 \mathrm{~min}, t_{\mathrm{R}}(\operatorname{minor})=12.8 \mathrm{~min} ; 94 \%$ ee.

$[\alpha]_{\mathrm{D}}^{25}=-80.8\left(c=1.8, \mathrm{CH}_{2} \mathrm{Cl}_{2}\right)$.

HRMS (ESI) calcd for [M+Na] $\mathrm{C}_{16} \mathrm{H}_{17} \mathrm{NO}_{6} \mathrm{Na}, \mathrm{m} / \mathrm{z}$ 342.0954, found 342.0956.

$(1 R, 5 S, 6 R, 7 S)$-methyl 5-hydroxy-7-(3-methoxyphenyl)-6-nitro-2-oxobic

yclo[3.2.1]octane-1-carboxylate (3b)

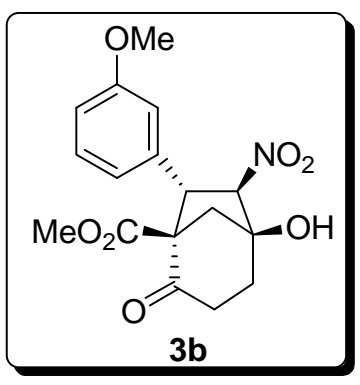

The title compound was prepared according to the typical procedure, as described above in $84 \%$ yield.

${ }^{1} \mathrm{H}-\mathrm{NMR}\left(400 \mathrm{MHz}, \mathrm{CDCl}_{3}\right) \delta$ 7.26-7.22 (m, 1H), 6.83-6.77 (m, 3H), $5.27(\mathrm{~d}, J=5.2 \mathrm{~Hz}$, 1H), $4.86(\mathrm{~d}, J=5.6 \mathrm{~Hz}, 1 \mathrm{H}), 3.85(\mathrm{~s}, 3 \mathrm{H}), 3.77(\mathrm{~s}, 3 \mathrm{H}), 3.00(\mathrm{dd}, J=3.6,12.8 \mathrm{~Hz}, 1 \mathrm{H})$, 2.59-2.52 (m, 2H), 2.26-2.77 (m, 3H).

${ }^{13} \mathrm{C}-\mathrm{NMR}\left(100 \mathrm{MHz}, \mathrm{CDCl}_{3}\right) \delta 203.5,169.8,159.8,137.3,130.0,119.2,113.4,113.0,98.5$, $76.2,65.0,55.2,53.0,52.2,44.8,37.0,35.2$.

HPLC: Chiralpak AD-H (hexane $/ i-\mathrm{PrOH}=85 / 15$, flow rate $1 \mathrm{~mL} / \min , \lambda=210 \mathrm{~nm}$ ), $t_{\mathrm{R}}$ $($ major $)=11.1 \min , t_{\mathrm{R}}($ minor $)=16.8 \mathrm{~min} ; 95 \%$ ee.

$[\alpha]_{\mathrm{D}}^{25}=-88.3\left(c=1.0, \mathrm{CH}_{2} \mathrm{Cl}_{2}\right)$.

HRMS (ESI) calcd for $[\mathrm{M}+\mathrm{Na}] \mathrm{C}_{17} \mathrm{H}_{19} \mathrm{NO}_{7} \mathrm{Na}, \mathrm{m} / \mathrm{z} 370.1059$, found 370.1054 .

$(1 R, 5 S, 6 R, 7 S)$-methyl 5-hydroxy-7-(4-methoxyphenyl)-6-nitro-2-oxobic yclo[3.2.1] octane-1-carboxylate (3c) 


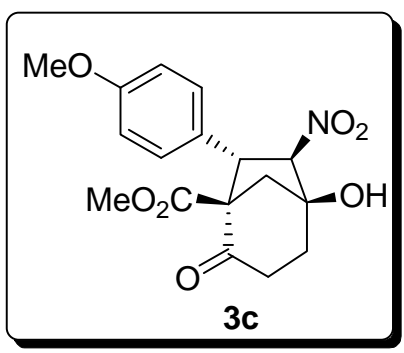

The title compound was prepared according to the typical procedure, as described above in $79 \%$ yield.

${ }^{1} \mathrm{H}-\mathrm{NMR}\left(400 \mathrm{MHz}, \mathrm{CDCl}_{3}\right) \delta 7.13(\mathrm{~d}, J=8.8 \mathrm{~Hz}, 2 \mathrm{H}), 6.85(\mathrm{~d}, J=8.4 \mathrm{~Hz}, 2 \mathrm{H}), 5.23(\mathrm{~d}, J$ $=5.6 \mathrm{~Hz}, 1 \mathrm{H}), 4.81(\mathrm{~d}, J=5.6 \mathrm{~Hz}, 1 \mathrm{H}), 3.84(\mathrm{~s}, 3 \mathrm{H}), 3.78(\mathrm{~s}, 3 \mathrm{H}), 2.98(\mathrm{dd}, J=3.6,12.8$ $\mathrm{Hz}, 1 \mathrm{H}), 2.96$ (brs, 1H), $2.58(\mathrm{dd}, J=6.8,16.8 \mathrm{~Hz}, 1 \mathrm{H}), 2.53(\mathrm{~d}, J=12.4 \mathrm{~Hz}, 1 \mathrm{H})$, 2.26-2.07 (m, 3H).

${ }^{13} \mathrm{C}-\mathrm{NMR}\left(100 \mathrm{MHz}, \mathrm{CDCl}_{3}\right) \delta 203.7,169.9,158.9,128.4,127.4,114.3,98.7,76.2,65.2$, $55.3,53.0,51.8,44.6,36.7,35.2$.

HPLC: Chiralpak AD-H (hexane $/ i-\mathrm{PrOH}=85 / 15$, flow rate $1 \mathrm{~mL} / \mathrm{min}, \lambda=210 \mathrm{~nm}$ ), $t_{\mathrm{R}}$ $($ major $)=13.9 \min , t_{\mathrm{R}}(\operatorname{minor})=18.7 \mathrm{~min} ; 90 \%$ ee.

$[\alpha]_{\mathrm{D}}^{25}=-76.0\left(c=1.5, \mathrm{CH}_{2} \mathrm{Cl}_{2}\right)$.

HRMS (ESI) calcd for $[\mathrm{M}+\mathrm{Na}] \mathrm{C}_{17} \mathrm{H}_{19} \mathrm{NO}_{7} \mathrm{Na}, \mathrm{m} / \mathrm{z} 370.1059$, found 370.1055 .

$(1 R, 5 S, 6 R, 7 S)$-methyl 5-hydroxy-6-nitro-2-oxo-7-p-tolylbicyclo[3.2.1]oc tane-1-carboxylate $(\mathbf{3 d})$

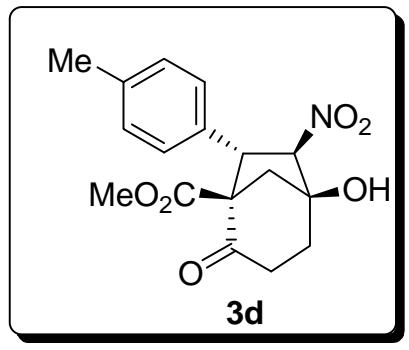

The title compound was prepared according to the typical procedure, as described above in 
$91 \%$ yield.

${ }^{1} \mathrm{H}-\mathrm{NMR}\left(400 \mathrm{MHz}, \mathrm{CDCl}_{3}\right) \delta 7.13(\mathrm{~d}, J=8.4 \mathrm{~Hz}, 2 \mathrm{H}), 7.08(\mathrm{~d}, J=8.4 \mathrm{~Hz}, 2 \mathrm{H}), 5.23(\mathrm{dd}$, $J=0.4,5.6 \mathrm{~Hz}, 1 \mathrm{H}), 4.81(\mathrm{~d}, J=5.2 \mathrm{~Hz}, 1 \mathrm{H}), 3.84(\mathrm{~s}, 3 \mathrm{H}), 3.00(\mathrm{dd}, J=3.6,12.4 \mathrm{~Hz}, 1 \mathrm{H})$, 2.91 (brs, 1H), 2.58-2.51 (m, 3H), $2.32(\mathrm{~s}, 3 \mathrm{H}), 2.26-2.11(\mathrm{~m}, 3 \mathrm{H})$.

${ }^{13} \mathrm{C}-\mathrm{NMR}\left(100 \mathrm{MHz}, \mathrm{CDCl}_{3}\right) \delta 203.6,169.8,137.5,132.6,129.6,127.0,98.7,76.2,65.0$, 53.0, 52.1, 44.7, 37.0, 35.2, 20.9 .

HPLC: Chiralpak AD-H (hexane / $i-\mathrm{PrOH}=85 / 15$, flow rate $1 \mathrm{~mL} / \min , \lambda=210 \mathrm{~nm}$ ), $t_{\mathrm{R}}$ $($ major $)=9.7 \mathrm{~min}, t_{\mathrm{R}}(\operatorname{minor})=12.4 \mathrm{~min} ; 94 \%$ ee.

$[\alpha]_{\mathrm{D}}^{25}=-94.4\left(c=0.9, \mathrm{CH}_{2} \mathrm{Cl}_{2}\right)$.

HRMS (ESI) calcd for $[\mathrm{M}+\mathrm{Na}] \mathrm{C}_{17} \mathrm{H}_{19} \mathrm{NO}_{6} \mathrm{Na}, \mathrm{m} / \mathrm{z} 356.1110$, found 356.1106.

(1R,5S,6R,7S)-methyl 7-(4-bromophenyl)-5-hydroxy-6-nitro-2-oxobicycl

o[3.2.1]octane-1-carboxylate (3e)

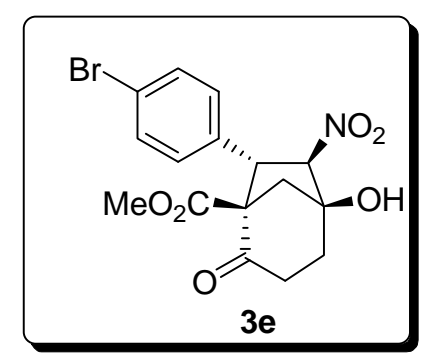

The title compound was prepared according to the typical procedure, as described above in $81 \%$ yield.

${ }^{1} \mathrm{H}-\mathrm{NMR}\left(400 \mathrm{MHz}, \mathrm{CDCl}_{3}\right) \delta 7.46(\mathrm{~d}, J=8.8 \mathrm{~Hz}, 2 \mathrm{H}), 7.09(\mathrm{~d}, J=8.0 \mathrm{~Hz}, 2 \mathrm{H}), 5.20(\mathrm{~d}, J$ $=5.6 \mathrm{~Hz}, 1 \mathrm{H}), 4.80(\mathrm{~d}, J=5.2 \mathrm{~Hz}, 1 \mathrm{H}), 3.85(\mathrm{~s}, 3 \mathrm{H}), 2.99(\mathrm{dd}, J=3.6,12.8 \mathrm{~Hz}, 1 \mathrm{H}), 2.81$ (brs, 1H), 2.63-2.53 (m, 2H), 2.31-2.10 (m, 2H), 2.07-2.00 (m, 1H).

${ }^{13} \mathrm{C}-\mathrm{NMR}\left(100 \mathrm{MHz}, \mathrm{CDCl}_{3}\right) \delta 203.2,169.7,134.7,132.1,129.0,122.0,98.7,76.2,65.0$, $53.1,51.8,44.6,36.9,35.2$. 
HPLC: Chiralpak AD-H (hexane / $i-\mathrm{PrOH}=85 / 15$, flow rate $1 \mathrm{~mL} / \mathrm{min}, \lambda=210 \mathrm{~nm}$ ), $t_{\mathrm{R}}$ $($ major $)=11.1 \mathrm{~min}, t_{\mathrm{R}}(\operatorname{minor})=14.6 \mathrm{~min} ; 94 \%$ ee.

$[\alpha]_{\mathrm{D}}^{25}=-70.3\left(c=1.2, \mathrm{CH}_{2} \mathrm{Cl}_{2}\right)$.

HRMS (ESI) calcd for $[\mathrm{M}+\mathrm{Na}] \mathrm{C}_{16} \mathrm{H}_{16} \mathrm{NO}_{6} \mathrm{NaBr}, \mathrm{m} / \mathrm{z} 420.0059$, found 420.0046.

$(1 R, 5 S, 6 R, 7 R)$-methyl

7-(2-chlorophenyl)-5-hydroxy-6-nitro-2-oxobicycl

$\mathrm{o}[3.2 .1]$ octane-1-carboxylate (3f)

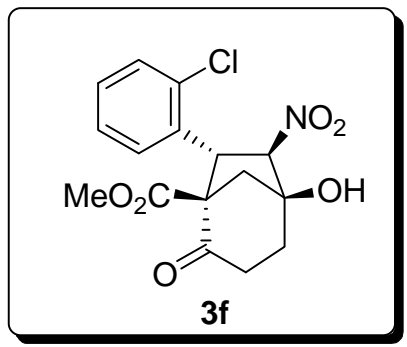

The title compound was prepared according to the typical procedure, as described above in $86 \%$ yield.

${ }^{1} \mathrm{H}-\mathrm{NMR}\left(400 \mathrm{MHz}, \mathrm{CDCl}_{3}\right) \delta 7.43(\mathrm{~d}, J=8.0 \mathrm{~Hz}, 1 \mathrm{H}), 7.27-7.18(\mathrm{~m}, 2 \mathrm{H}), 7.13(\mathrm{~d}, J=8.0$ $\mathrm{Hz}, 1 \mathrm{H}), 5.14(\mathrm{~d}, J=6.0 \mathrm{~Hz}, 1 \mathrm{H}), 5.08(\mathrm{~d}, J=6.0 \mathrm{~Hz}, 1 \mathrm{H}), 3.76(\mathrm{~s}, 3 \mathrm{H}), 3.00(\mathrm{dd}, J=3.2$, $12.8 \mathrm{~Hz}, 1 \mathrm{H}), 2.96$ (brs, 1H), 2.81-2.76 (m, 1H), 2.60-2.49 (m, 2H), 2.35-2.25 (m, 2H).

${ }^{13} \mathrm{C}-\mathrm{NMR}\left(100 \mathrm{MHz}, \mathrm{CDCl}_{3}\right) \delta 205.2,169.0,134.8,133.6,130.8,129.3,128.1,127.0$, 97.7, 76.2, 64.8, 52.9, 50.9, 44.0, 36.8, 35.6.

HPLC: Chiralpak AD-H (hexane $/ i-\mathrm{PrOH}=85 / 15$, flow rate $1 \mathrm{~mL} / \min , \lambda=210 \mathrm{~nm}$ ), $t_{\mathrm{R}}$ $($ major $)=11.5 \mathrm{~min}, t_{\mathrm{R}}($ minor $)=21.8 \mathrm{~min} ; 96 \%$ ee.

$[\alpha]_{\mathrm{D}}^{25}=-81.7\left(c=0.4, \mathrm{CH}_{2} \mathrm{Cl}_{2}\right)$.

HRMS (ESI) calcd for [M+Na] $\mathrm{C}_{16} \mathrm{H}_{16} \mathrm{NO}_{6} \mathrm{NaCl}, \mathrm{m} / \mathrm{z} 376.0564$, found 376.0558. 
o[3.2.1]octane-1-carboxylate (3g)

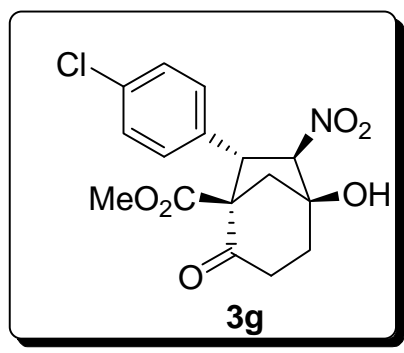

The title compound was prepared according to the typical procedure, as described above in $93 \%$ yield.

${ }^{1} \mathrm{H}-\mathrm{NMR}\left(400 \mathrm{MHz}, \mathrm{CDCl}_{3}\right) \delta 7.29(\mathrm{~d}, J=8.8 \mathrm{~Hz}, 2 \mathrm{H}), 7.16(\mathrm{~d}, J=8.4 \mathrm{~Hz}, 2 \mathrm{H}), 5.20(\mathrm{~d}, J$ $=5.6 \mathrm{~Hz}, 1 \mathrm{H}), 4.82(\mathrm{~d}, J=5.2 \mathrm{~Hz}, 1 \mathrm{H}), 3.85(\mathrm{~s}, 3 \mathrm{H}), 2.99(\mathrm{dd}, J=3.6,12.8 \mathrm{~Hz}, 1 \mathrm{H}), 2.76$ (brs, 1H), 2.64-2.54 (m, 2H), 2.32-2.18 (m, 2H), 2.10-2.06 (m, 1H).

${ }^{13} \mathrm{C}-\mathrm{NMR}\left(100 \mathrm{MHz}, \mathrm{CDCl}_{3}\right) \delta 203.2,169.7,134.1,133.9,129.1,128.6,98.3,76.2,65.0$, $53.1,51.8,44.6,36.9,35.1$.

HPLC: Chiralpak AD-H (hexane / $i-\mathrm{PrOH}=85 / 15$, flow rate $1 \mathrm{~mL} / \mathrm{min}, \lambda=210 \mathrm{~nm}$ ), $t_{\mathrm{R}}$ $($ major $)=10.0 \mathrm{~min}, t_{\mathrm{R}}(\operatorname{minor})=13.6 \mathrm{~min} ; 95 \%$ ee.

$[\alpha]_{\mathrm{D}}^{25}=-95.3\left(c=0.9, \mathrm{CH}_{2} \mathrm{Cl}_{2}\right)$.

HRMS (ESI) calcd for [M+Na] $\mathrm{C}_{16} \mathrm{H}_{16} \mathrm{NO}_{6} \mathrm{NaCl}, \mathrm{m} / \mathrm{z}$ 376.0564, found 376.0556.

$(1 R, 5 S, 6 R, 7 S)$-methyl 7-(4-fluorophenyl)-5-hydroxy-6-nitro-2-oxobicycl $\mathrm{o}[3.2 .1]$ octane-1-carboxylate (3h)

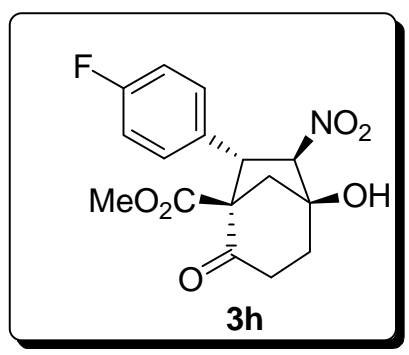

The title compound was prepared according to the typical procedure, as described above in 
$88 \%$ yield.

${ }^{1} \mathrm{H}-\mathrm{NMR}\left(400 \mathrm{MHz}, \mathrm{CDCl}_{3}\right) \delta$ 7.21-7.18 (m, 2H), 7.05-7.00 (m, 2H), $5.22(\mathrm{~d}, J=5.2 \mathrm{~Hz}$, $1 \mathrm{H}), 4.83(\mathrm{~d}, J=5.6 \mathrm{~Hz}, 1 \mathrm{H}), 3.85(\mathrm{~s}, 3 \mathrm{H}), 2.99(\mathrm{dd}, J=3.6,12.4 \mathrm{~Hz}, 1 \mathrm{H}), 2.91$ (brs, $1 \mathrm{H})$, 2.64-2.57 (m, 1H), $2.55(\mathrm{~d}, J=12.8 \mathrm{~Hz}, 1 \mathrm{H}), 2.29-2.22(\mathrm{~m}, 2 \mathrm{H}), 2.11-2.03(\mathrm{~m}, 1 \mathrm{H})$.

${ }^{13} \mathrm{C}-\mathrm{NMR}\left(100 \mathrm{MHz}, \mathrm{CDCl}_{3}\right) \delta 203.4,169.87,131.4,129.0,128.9,116.0,115.8,98.5$, $76.2,65.2,53.1,51.7,44.6,36.9,35.2$.

HPLC: Chiralpak AD-H (hexane / $i-\mathrm{PrOH}=85 / 15$, flow rate $1 \mathrm{~mL} / \min , \lambda=210 \mathrm{~nm}$ ), $t_{\mathrm{R}}$ $($ major $)=8.9 \min , t_{\mathrm{R}}(\operatorname{minor})=13.3 \mathrm{~min} ; 94 \%$ ee.

$[\alpha]_{\mathrm{D}}^{25}=-105.3\left(c=1.0, \mathrm{CH}_{2} \mathrm{Cl}_{2}\right)$.

HRMS (ESI) calcd for [M+Na] $\mathrm{C}_{16} \mathrm{H}_{16} \mathrm{NO}_{6} \mathrm{NaF}, \mathrm{m} / \mathrm{z} 360.0859$, found 360.0848 .

$(1 R, 5 S, 6 R, 7 S)$-methyl 5-hydroxy-6-nitro-2-oxo-7-(thiophen-2-yl)bicycle

[3.2.1] octane-1-carboxylate (3i)

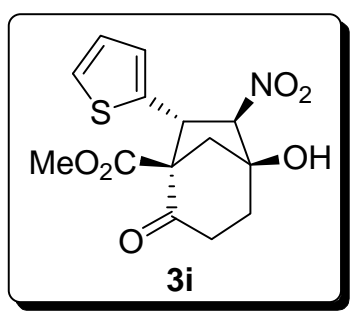

The title compound was prepared according to the typical procedure, as described above in $80 \%$ yield.

${ }^{1} \mathrm{H}-\mathrm{NMR}\left(400 \mathrm{MHz}, \mathrm{CDCl}_{3}\right) \delta 7.25(\mathrm{~d}, J=5.2 \mathrm{~Hz}, 1 \mathrm{H}), 7.04(\mathrm{~d}, J=3.2 \mathrm{~Hz}, 1 \mathrm{H}), 6.95(\mathrm{dd}$, $J=4.0,5.2 \mathrm{~Hz}, 1 \mathrm{H}), 5.38(\mathrm{~d}, J=5.2 \mathrm{~Hz}, 1 \mathrm{H}), 5.02(\mathrm{~d}, J=5.2 \mathrm{~Hz}, 1 \mathrm{H}), 3.87(\mathrm{~s}, 3 \mathrm{H})$, 2.96-2.93 (m, 2H), 2.91 (brs, 1H), 2.64-2.53 (m, 2H), 2.32-2.27 (m, 3H).

${ }^{13} \mathrm{C}-\mathrm{NMR}\left(100 \mathrm{MHz}, \mathrm{CDCl}_{3}\right) \delta 202.2,169.5,137.4,127.3,126.7,125.6,97.8,76.4,66.3$, $53.0,48.9,44.1,36.8,35.0$. 
HPLC: Chiralpak AD-H (hexane / $i-\mathrm{PrOH}=85 / 15$, flow rate $1 \mathrm{~mL} / \mathrm{min}, \lambda=210 \mathrm{~nm}$ ), $t_{\mathrm{R}}$ $($ major $)=10.3 \mathrm{~min}, t_{\mathrm{R}}($ minor $)=15.0 \mathrm{~min} ; 92 \%$ ee.

$[\alpha]_{\mathrm{D}}^{25}=-116.8\left(c=1.0, \mathrm{CH}_{2} \mathrm{Cl}_{2}\right)$.

HRMS (ESI) calcd for [M+Na] $\mathrm{C}_{14} \mathrm{H}_{15} \mathrm{NO}_{6} \mathrm{NaS}, \mathrm{m} / \mathrm{z} 348.0518$, found 348.0523.

$(1 R, 5 S, 6 R, 7 S)$-methyl

7-(furan-3-yl)-5-hydroxy-6-nitro-2-oxobicyclo

[3.2.1] octane-1-carboxylate (3j)

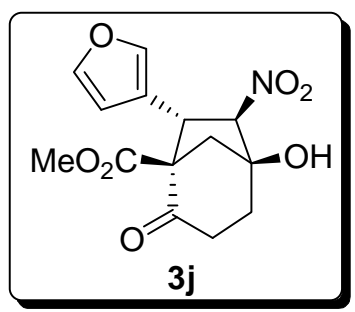

The title compound was prepared according to the typical procedure, as described above in $84 \%$ yield.

${ }^{1} \mathrm{H}-\mathrm{NMR}\left(400 \mathrm{MHz}, \mathrm{CDCl}_{3}\right) \delta 7.34(\mathrm{~s}, 1 \mathrm{H}), 6.34-6.31(\mathrm{~m}, 2 \mathrm{H}), 5.43(\mathrm{~d}, J=5.6 \mathrm{~Hz}, 1 \mathrm{H})$, $4.80(\mathrm{~d}, J=5.2 \mathrm{~Hz}, 1 \mathrm{H}), 3.82(\mathrm{~s}, 3 \mathrm{H}), 2.86(\mathrm{~d}, J=12.8 \mathrm{~Hz}, 1 \mathrm{H}), 2.80$ (brs, $1 \mathrm{H}), 2.67-2.49$ $(\mathrm{m}, 3 \mathrm{H}), 2.31-2.26(\mathrm{~m}, 2 \mathrm{H})$.

${ }^{13} \mathrm{C}-\mathrm{NMR}\left(100 \mathrm{MHz}, \mathrm{CDCl}_{3}\right) \delta 202.6,169.1,146.8,142.4,111.1,109.7,94.4,76.4,64.8$, $52.8,47.4,43.5,36.7,34.3$.

HPLC: Chiralpak AD-H (hexane $/ i-\mathrm{PrOH}=85 / 15$, flow rate $1 \mathrm{~mL} / \mathrm{min}, \lambda=210 \mathrm{~nm}$ ), $t_{\mathrm{R}}$ $($ major $)=9.1 \mathrm{~min}, t_{\mathrm{R}}(\operatorname{minor})=12.3 \mathrm{~min} ; 94 \%$ ee.

$[\alpha]_{\mathrm{D}}^{25}=-85.9\left(c=1.5, \mathrm{CH}_{2} \mathrm{Cl}_{2}\right)$.

HRMS (ESI) calcd for $[\mathrm{M}+\mathrm{Na}] \mathrm{C}_{14} \mathrm{H}_{15} \mathrm{NO}_{7} \mathrm{Na}, \mathrm{m} / \mathrm{z} 332.0746$, found 332.0753 . 
lo[3.2.1] octane-1-carboxylate (3k)

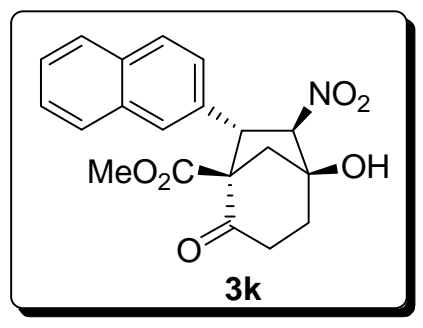

The title compound was prepared according to the typical procedure, as described above in $85 \%$ yield.

${ }^{1} \mathrm{H}-\mathrm{NMR}\left(400 \mathrm{MHz}, \mathrm{CDCl}_{3}\right) \delta$ 7.82-7.78 (m, 3H), $7.67(\mathrm{~s}, 1 \mathrm{H}), 7.49(\mathrm{dd}, J=3.2,6.0 \mathrm{~Hz}$, 1H), 7.30-7.28 (m, 1H), $5.36(\mathrm{~d}, J=5.6 \mathrm{~Hz}, 1 \mathrm{H}), 5.04(\mathrm{~d}, J=5.6 \mathrm{~Hz}, 1 \mathrm{H}), 3.87(\mathrm{~s}, 3 \mathrm{H})$, $3.06(\mathrm{dd}, J=3.2,12.4 \mathrm{~Hz}, 1 \mathrm{H}), 2.90$ (brs, $1 \mathrm{H}), 2.59-2.51(\mathrm{~m}, 2 \mathrm{H}), 2.30-2.06(\mathrm{~m}, 3 \mathrm{H})$.

${ }^{13} \mathrm{C}-\mathrm{NMR}\left(100 \mathrm{MHz}, \mathrm{CDCl}_{3}\right) \delta 203.7,169.9,133.1,133.0,132.4,128.8,128.1,127.5$, $126.7,126.6,126.1,124.9,98.6,76.3,65.1,53.1,52.4,44.8,37.0,35.3$.

HPLC: Chiralpak AD-H (hexane / $i-\mathrm{PrOH}=85 / 15$, flow rate $1 \mathrm{~mL} / \min , \lambda=254 \mathrm{~nm}$ ), $t_{\mathrm{R}}$ $($ major $)=11.2 \mathrm{~min}, t_{\mathrm{R}}(\operatorname{minor})=21.0 \mathrm{~min} ; 94 \%$ ee.

$[\alpha]_{\mathrm{D}}^{25}=-77.7\left(c=1.2, \mathrm{CH}_{2} \mathrm{Cl}_{2}\right)$.

HRMS (ESI) calcd for $[\mathrm{M}+\mathrm{Na}] \mathrm{C}_{20} \mathrm{H}_{19} \mathrm{NO}_{6} \mathrm{Na}, \mathrm{m} / \mathrm{z}$ 392.1110, found 392.1107.

$(1 R, 5 S, 6 R, 7 S)$-methyl 5-hydroxy-6-nitro-7-(4-nitrophenyl)-2-oxobicyclo

[3.2.1] octane-1-carboxylate (3I)

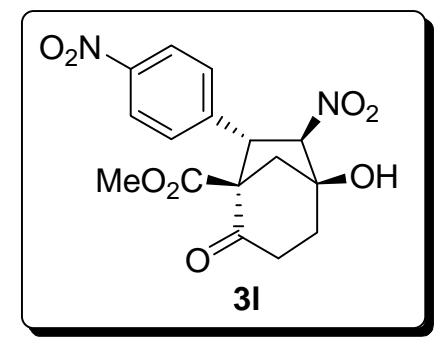

The title compound was prepared according to the typical procedure, as described above in 
$77 \%$ yield.

${ }^{1} \mathrm{H}-\mathrm{NMR}\left(400 \mathrm{MHz}, \mathrm{CDCl}_{3}\right) \delta 8.19(\mathrm{~d}, J=8.8 \mathrm{~Hz}, 2 \mathrm{H}), 7.41(\mathrm{~d}, J=9.2 \mathrm{~Hz}, 2 \mathrm{H}), 5.25(\mathrm{~d}, J$ $=6.0 \mathrm{~Hz}, 1 \mathrm{H}), 4.93(\mathrm{~d}, J=5.6 \mathrm{~Hz}, 1 \mathrm{H}), 3.86(\mathrm{~s}, 3 \mathrm{H}), 3.00(\mathrm{dd}, J=3.6,12.8 \mathrm{~Hz}, 1 \mathrm{H}), 2.96$ (brs, 1H), 2.68-2.58 (m, 2H), 2.36-2.21 (m, 2H), 2.06-1.99 (m, 1H).

${ }^{13} \mathrm{C}-\mathrm{NMR}\left(100 \mathrm{MHz}, \mathrm{CDCl}_{3}\right) \delta 202.8,169.5,147.3,143.0,128.4,124.0,97.8,76.3,65.1$, $53.3,52.0,44.3,36.8,35.1$.

HPLC: Chiralpak AD-H (hexane / $i-\mathrm{PrOH}=85 / 15$, flow rate $1 \mathrm{~mL} / \min , \lambda=254 \mathrm{~nm}$ ), $t_{\mathrm{R}}$ $($ major $)=20.2 \min , t_{\mathrm{R}}($ minor $)=27.8 \min ; 94 \%$ ee.

$[\alpha]_{\mathrm{D}}^{25}=-96.2\left(c=0.7, \mathrm{CH}_{2} \mathrm{Cl}_{2}\right)$.

HRMS (ESI) calcd for [M+Na] $\mathrm{C}_{16} \mathrm{H}_{16} \mathrm{~N}_{2} \mathrm{O}_{8} \mathrm{Na}, \mathrm{m} / \mathrm{z} 387.0804$, found 387.0807.

$(1 R, 5 S, 6 R, 7 S)$-methyl 5-hydroxy-6-nitro-2-oxo-7-styrylbicyclo[3.2.1]oct

ane-1-carboxylate $(3 \mathbf{m})$

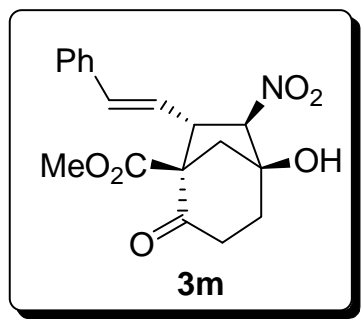

The title compound was prepared according to the typical procedure, as described above in $73 \%$ yield.

${ }^{1} \mathrm{H}-\mathrm{NMR}\left(400 \mathrm{MHz}, \mathrm{CDCl}_{3}\right) \delta$ 7.37-7.28 (m, 5H), $6.67(\mathrm{~d}, J=16.8 \mathrm{~Hz}, 1 \mathrm{H}), 6.30(\mathrm{dd}, J=$ 4.8, $12.0 \mathrm{~Hz}, 1 \mathrm{H}), 5.14(\mathrm{~d}, J=5.2 \mathrm{~Hz}, 1 \mathrm{H}), 4.26-4.22(\mathrm{~m}, 1 \mathrm{H}), 3.85(\mathrm{~s}, 3 \mathrm{H}), 2.89-2.86(\mathrm{~m}$, 1H), 2.79 (brs, 1H), 2.70-2.65 (m, 1H), 2.49 (d, $J=12.4 \mathrm{~Hz}, 1 \mathrm{H}), 2.39-2.24$ (m, 3H).

${ }^{13} \mathrm{C}-\mathrm{NMR}\left(100 \mathrm{MHz}, \mathrm{CDCl}_{3}\right) \delta 202.5,166.9,149.9,140.0,129.6,129.2,128.5,126.1$, $121.1,99.9,78.1,77.265 .3,49.1,44.4,34.2,33.0$. 
HPLC: Chiralpak AD-H (hexane / $i-\mathrm{PrOH}=85 / 15$, flow rate $1 \mathrm{~mL} / \min , \lambda=254 \mathrm{~nm}$ ), $t_{\mathrm{R}}$ $($ major $)=10.3 \min , t_{\mathrm{R}}($ minor $)=14.2 \mathrm{~min} ; 86 \%$ ee.

$[\alpha]_{\mathrm{D}}^{25}=-37.6\left(c=1.5, \mathrm{CH}_{2} \mathrm{Cl}_{2}\right)$.

HRMS (ESI) calcd for [M+Na] $\mathrm{C}_{18} \mathrm{H}_{19} \mathrm{NO}_{6} \mathrm{Na}, \mathrm{m} / \mathrm{z} 368.1110$, found 332.1113.

$(1 R, 5 S, 6 R, 7 S)$-phenyl

5-hydroxy-6-nitro-2-oxo-7-phenylbicyclo[3.2.1]oc

tane-1-carboxylate (3n)

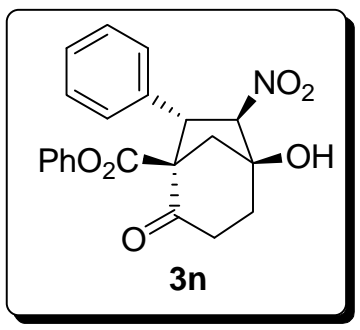

Catalyst VI $(0.005$ mmol, 0.05 eq $)$ was added to a solution of phenyl 2,5-dioxocyclohexanecarboxylate $\mathbf{1 b}(0.2 \mathrm{mmol}, 2 \mathrm{eq})$ and nitroolefin $\mathbf{2 a}(0.1 \mathrm{mmol}, 1 \mathrm{eq})$ in diethyl ether $(0.5 \mathrm{~mL})$ at room temperature. The resulting mixture was stirred vigorously. After the reaction was complete (monitored by TLC and crude NMR), the products were afforded by flash chromatography over silica gel (EtOAc : Hexane $=1: 4$ to $1: 2)$.

${ }^{1} \mathrm{H}-\mathrm{NMR}\left(400 \mathrm{MHz}, \mathrm{CDCl}_{3}\right) \delta$ 7.44-7.38 (m, 5H), 7.22-7.13 (m, 3H), $6.327(\mathrm{~d}, J=7.6 \mathrm{~Hz}$, 2H), $5.34(\mathrm{~d}, J=6.8 \mathrm{~Hz}, 1 \mathrm{H}), 4.58(\mathrm{~d}, J=6.8 \mathrm{~Hz}, 1 \mathrm{H}), 3.26(\mathrm{dd}, J=3.6,13.6 \mathrm{~Hz}, 1 \mathrm{H})$, 3.14 (brs, 1H), 3.11-3.02 (m, 1H), 2.68 (dd, $J=8.0,16.8 \mathrm{~Hz}, 1 \mathrm{H}), 2.55(\mathrm{~d}, J=13.2 \mathrm{~Hz}$, $1 \mathrm{H}), 2.30-2.14(\mathrm{~m}, 2 \mathrm{H})$.

${ }^{13} \mathrm{C}-\mathrm{NMR}\left(100 \mathrm{MHz}, \mathrm{CDCl}_{3}\right) \delta 202.5,169.5,135.6,134.0,128.7,128.4,126.6,123.1$, $95.0,76.6,65.0,52.8,50.4,43.1,36.8,34.7$. 
HPLC: Chiralpak AD-H (hexane / $i-\mathrm{PrOH}=85 / 15$, flow rate $1 \mathrm{~mL} / \mathrm{min}, \lambda=210 \mathrm{~nm}$ ), $t_{\mathrm{R}}$ $($ major $)=12.4 \min , t_{\mathrm{R}}(\operatorname{minor})=19.7 \mathrm{~min} ; 85 \%$ ee.

$[\alpha]_{\mathrm{D}}^{25}=-79.5\left(c=1.4, \mathrm{CH}_{2} \mathrm{Cl}_{2}\right)$.

HRMS (ESI) calcd for [M+Na] $\mathrm{C}_{21} \mathrm{H}_{19} \mathrm{NO}_{6} \mathrm{Na}, \mathrm{m} / \mathrm{z}$ 404.1110, found 404.1104.

General procedure for the preparation of $(1 \mathrm{a})$ and $(1 \mathbf{b})$<smiles>O=C1CCC(=O)CC1</smiles>

NaHMDS (20 mmol, $2 \mathrm{M}$ in THF) was slowly added to solution of cyclohexane-1,4-dione (20 mmol) in dry THF $(30 \mathrm{~mL})$ at $-78^{\circ} \mathrm{C}$. After $30 \mathrm{~min}$ stirring at the same temperature, a solution of methyl or phenyl chloroformate $(20 \mathrm{mmol})$ was added, and the resulting mixture was stirred for $1 \mathrm{~h}$ at $-78^{\circ} \mathrm{C}$, and then allowed to warm to room temperature. The reaction was then quenched by addition of sat. $\mathrm{NH}_{4} \mathrm{Cl}$ (aq.), and the product extracted with $\mathrm{Et}_{2} \mathrm{O}(3 \times 30 \mathrm{~mL})$. The combined organic phases were then dried over $\mathrm{Na}_{2} \mathrm{SO}_{4}$, filtered and evaporated under reduced pressure. Purification of the crude mixture by FC (ethyl acetate in $n$-hexane 1:8), gave the corresponding $\beta$-ketoesters $\mathbf{1 a}$ or $\mathbf{1 b}$.

Methyl 2,5-dioxocyclohexanecarboxylate (1a)

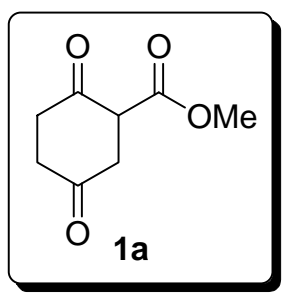

The title compound was obtained according to the general procedure as colorless oil $(15 \%$ 
yield).

${ }^{1} \mathrm{H}-\mathrm{NMR}\left(400 \mathrm{MHz}, \mathrm{CDCl}_{3}\right) \delta 12.21(\mathrm{~s}, 1 \mathrm{H}), 3.79(\mathrm{~s}, 3 \mathrm{H}), 3.10(\mathrm{~s}, 2 \mathrm{H}), 2.71(\mathrm{t}, J=6.8 \mathrm{~Hz}$, 2H), $2.59(\mathrm{t}, J=6.8 \mathrm{~Hz}, 2 \mathrm{H})$. (Almost $\mathbf{1 0 0 \%}$ enol form)

${ }^{13} \mathrm{C}-\mathrm{NMR}\left(100 \mathrm{MHz}, \mathrm{CDCl}_{3}\right) \delta 207.5,171.7,170.7,94.5,51.8,37.2,36.6,28.3$.

HRMS (ESI) calcd for $[\mathrm{M}+\mathrm{Na}] \mathrm{C}_{8} \mathrm{H}_{10} \mathrm{O}_{4} \mathrm{Na}, \mathrm{m} / \mathrm{z}$ 193.0477, found 193.0477.

Phenyl 2,5-dioxocyclohexanecarboxylate (1b)

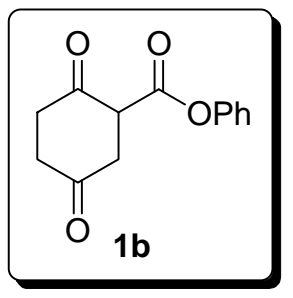

The title compound was obtained according to the general procedure as colorless oil $(20 \%$ yield).

${ }^{1} \mathrm{H}-\mathrm{NMR}\left(400 \mathrm{MHz}, \mathrm{CDCl}_{3}\right) \delta 12.06(\mathrm{~s}, 1 \mathrm{H}), 7.43(\mathrm{t}, J=8.0 \mathrm{~Hz}, 2 \mathrm{H}), 7.31-7.27(\mathrm{~m}, 1 \mathrm{H})$, $7.13(\mathrm{~d}, J=8.0 \mathrm{~Hz}, 2 \mathrm{H}), 3.34(\mathrm{~s}, 2 \mathrm{H}), 2.79(\mathrm{t}, J=6.8 \mathrm{~Hz}, 2 \mathrm{H}), 2.66(\mathrm{t}, J=6.8 \mathrm{~Hz}, 2 \mathrm{H})$. (Almost $100 \%$ enol form)

${ }^{13} \mathrm{C}-\mathrm{NMR}\left(100 \mathrm{MHz}, \mathrm{CDCl}_{3}\right) \delta 207.0,172.9,170.0,150.0,129.6,126.2,121.6,94.2,37.1$, $36.7,28.6$.

HRMS (ESI) calcd for $[\mathrm{M}+\mathrm{H}] \mathrm{C}_{13} \mathrm{H}_{14} \mathrm{O}_{4}, \mathrm{~m} / \mathrm{z} 233.0814$, found 233.0812 . 


\section{DFT calculation}

TSI-1(S,S)

\begin{tabular}{|c|c|c|c|}
\hline $\mathrm{C}$ & 0.86070 & 2.80113 & 1.22678 \\
\hline $\mathrm{C}$ & 1.01950 & 2.96015 & 3.73649 \\
\hline $\mathrm{C}$ & 0.22158 & 3.40800 & 2.49451 \\
\hline $\mathrm{H}$ & 1.65216 & 3.46183 & 0.86992 \\
\hline $\mathrm{H}$ & 0.22873 & 4.49962 & 2.39968 \\
\hline $\mathrm{H}$ & -0.82899 & 3.10714 & 2.56674 \\
\hline $\mathrm{C}$ & 2.89612 & 2.03066 & 2.35261 \\
\hline $\mathrm{H}$ & 3.37577 & 1.13749 & 2.75352 \\
\hline $\mathrm{H}$ & 3.53432 & 2.43010 & 1.56176 \\
\hline $\mathrm{C}$ & 2.52792 & 3.07782 & 3.42991 \\
\hline $\mathrm{H}$ & 3.11981 & 2.89753 & 4.33344 \\
\hline $\mathrm{H}$ & 2.76599 & 4.09088 & 3.08613 \\
\hline $\mathrm{H}$ & 0.75534 & 3.57162 & 4.60429 \\
\hline $\mathrm{C}$ & 0.71925 & 1.46491 & 4.02972 \\
\hline $\mathrm{H}$ & 1.48612 & 1.09895 & 4.72459 \\
\hline $\mathrm{C}$ & 0.84715 & 0.70908 & 2.68223 \\
\hline $\mathrm{H}$ & 1.39373 & -0.22919 & 2.76523 \\
\hline $\mathrm{H}$ & -0.12400 & 0.50408 & 2.23936 \\
\hline $\mathrm{N}$ & 1.62223 & 1.55349 & 1.68716 \\
\hline & -0.62672 & 1.22159 & 4.66235 \\
\hline
\end{tabular}




$\begin{array}{llll}\mathrm{H} & -1.49987 & 1.50593 & 4.07407 \\ \mathrm{C} & -0.80264 & 0.68597 & 5.87088 \\ \mathrm{H} & -1.79410 & 0.53514 & 6.28825 \\ \mathrm{H} & 0.03771 & 0.37544 & 6.48905 \\ \mathrm{C} & -0.14131 & 2.63285 & 0.04409 \\ \mathrm{~N} & -0.94431 & 1.43805 & 0.15849 \\ \mathrm{H} & -0.45995 & 0.53221 & 0.08438 \\ \mathrm{C} & -2.31313 & 1.40815 & 0.05569 \\ \mathrm{~S} & -3.27947 & 2.76515 & 0.31265 \\ \mathrm{~N} & -2.73954 & 0.14693 & -0.27091 \\ \mathrm{H} & -2.00486 & -0.47102 & -0.63506 \\ \mathrm{C} & -4.01612 & -0.43459 & -0.30678 \\ \mathrm{C} & -4.10628 & -1.67005 & -0.97426 \\ \mathrm{C} & -5.16226 & 0.09389 & 0.30324 \\ \mathrm{C} & -5.31774 & -2.35063 & -1.03743 \\ \mathrm{H} & -5.37605 & -3.70169 & -1.69969 \\ \mathrm{H} & -3.22127 & -2.08936 & -1.44335 \\ \mathrm{C} & -6.36889 & -0.60493 & 0.22461 \\ \mathrm{C} & -5.11207 & 1.03744 & 0.82695 \\ \mathrm{C} & -6.46592 & -1.82414 & -0.44236 \\ \mathrm{C} & -7.40951 & -2.35268 & -0.49653 \\ \mathrm{H} & -6.90025 & 0.01109 & 0.83763 \\ \mathrm{H} & -0.00651\end{array}$




$\begin{array}{llll}\mathrm{C} & -7.31604 & 0.66582 & 1.98498 \\ \mathrm{C} & -8.53900 & -0.91916 & 1.12469 \\ \mathrm{C} & -4.45599 & -3.82555 & -2.68038 \\ \mathrm{C} & -5.14278 & -4.69935 & -0.81414 \\ \mathrm{C} & -6.58891 & -3.94067 & -2.24587 \\ \mathrm{H} & -0.84596 & 3.46697 & 0.15939 \\ \mathrm{C} & 0.52402 & 2.78299 & -1.32964 \\ \mathrm{C} & 1.08804 & 4.04456 & -1.72640 \\ \mathrm{C} & 0.56836 & 1.75054 & -2.23915 \\ \mathrm{C} & 1.72374 & 4.10924 & -3.01193 \\ \mathrm{C} & 1.03351 & 5.22388 & -0.94432 \\ \mathrm{C} & 1.20168 & 1.93693 & -3.49456 \\ \mathrm{H} & 0.11718 & 0.78702 & -2.02851 \\ \mathrm{C} & 2.30434 & 5.33252 & -3.42949 \\ \mathrm{H} & 1.78151 & 3.05384 & -3.87589 \\ \mathrm{C} & 1.60148 & 6.40903 & -1.38590 \\ \mathrm{H} & 0.51281 & 5.25353 & 0.00639 \\ \mathrm{H} & 1.22361 & 1.10972 & -4.20374 \\ \mathrm{H} & 2.25589 & 6.46179 & -2.64266 \\ \mathrm{H} & 2.78100 & 5.34863 & -4.40451 \\ \mathrm{H} & 2.70484 & 7.38311 & -2.99541 \\ \mathrm{H} & 1.97619 & 8.73937 & -0.96938\end{array}$




$\begin{array}{llll}\mathrm{H} & 1.48727 & 9.07606 & -1.89248 \\ \mathrm{H} & 1.73969 & 9.43609 & -0.16319 \\ \mathrm{H} & 3.06346 & 8.72030 & -1.12121 \\ \mathrm{O} & -0.60962 & -1.50184 & -1.56904 \\ \mathrm{O} & 0.33031 & -1.01455 & 0.36382 \\ \mathrm{~N} & 0.29873 & -1.72105 & -0.70274 \\ \mathrm{C} & 1.19990 & -2.71322 & -0.90127 \\ \mathrm{H} & 1.03513 & -3.28187 & -1.80392 \\ \mathrm{C} & 2.18006 & -2.98950 & 0.08844 \\ \mathrm{H} & 1.99051 & -2.45065 & 1.00960 \\ \mathrm{C} & 2.66817 & -4.37489 & 0.27255 \\ \mathrm{C} & 2.99408 & -4.80339 & 1.57282 \\ \mathrm{C} & 2.77774 & -5.30040 & -0.78092 \\ \mathrm{H} & 3.40549 & -6.11233 & 1.81504 \\ \mathrm{H} & 1.93084 & 0.94363 & 0.89732 \\ \mathrm{H} & 2.89835 & -4.10382 & 2.39918 \\ \mathrm{C} & 3.19833 & -6.60649 & -0.53993 \\ \mathrm{H} & 2.54841 & -5.00136 & -1.79842 \\ \mathrm{H} & 3.51095 & -7.01873 & 0.75777 \\ \mathrm{H} & 3.63878 & -6.42616 & 2.82888 \\ \mathrm{H} & 3.28296 & -7.30285 & -1.36909 \\ \mathrm{H} & 3.83381 & -8.03942 & 0.94306 \\ \mathrm{H} & 0.30807 & -0.32372\end{array}$




$\begin{array}{llll}\mathrm{C} & 3.61211 & -0.64342 & -0.83797 \\ \mathrm{C} & 4.00060 & -0.52546 & -2.30948 \\ \mathrm{C} & 4.02531 & -1.87906 & -0.14448 \\ \mathrm{C} & 3.91059 & -1.84579 & -3.08048 \\ \mathrm{H} & 3.37925 & 0.25356 & -2.75758 \\ \mathrm{C} & 5.01626 & -2.74821 & -0.92061 \\ \mathrm{C} & 4.67629 & -2.96569 & -2.39190 \\ \mathrm{H} & 2.86144 & -2.16246 & -3.15518 \\ \mathrm{H} & 6.01848 & -2.28656 & -0.90344 \\ \mathrm{C} & 4.31115 & -1.72114 & 1.29864 \\ \mathrm{O} & 3.64717 & -1.08848 & 2.11308 \\ \mathrm{O} & 5.41905 & -2.41065 & 1.67704 \\ \mathrm{C} & 5.74662 & -2.35182 & 3.07032 \\ \mathrm{H} & 4.92019 & -2.72567 & 3.68109 \\ \mathrm{H} & 6.62663 & -2.98466 & 3.18990 \\ \mathrm{H} & 5.97186 & -1.32483 & 3.37227 \\ \mathrm{H} & 5.03753 & -0.15508 & -2.33620 \\ \mathrm{H} & 4.27746 & -1.74807 & -4.10746 \\ \mathrm{O} & 5.14113 & -3.72681 & -0.45510 \\ \mathrm{H} & 5.01714 & -3.97123 & -2.98453\end{array}$

TSI-2 (R,S)
C
1.47694
2.47999
1.29665 


$\begin{array}{llll}\mathrm{C} & 1.71582 & 2.17316 & 3.78639 \\ \mathrm{C} & 0.94623 & 2.92143 & 2.67952 \\ \mathrm{H} & 2.33599 & 3.09811 & 1.03079 \\ \mathrm{H} & 1.08033 & 4.00435 & 2.77635 \\ \mathrm{H} & -0.12964 & 2.73147 & 2.75085 \\ \mathrm{C} & 3.42607 & 1.28841 & 2.17193 \\ \mathrm{H} & 3.80434 & 0.29039 & 2.38972 \\ \mathrm{H} & 4.09171 & 1.75187 & 1.43934 \\ \mathrm{C} & 3.21899 & 2.16228 & 3.43320 \\ \mathrm{H} & 3.80604 & 1.75265 & 4.26183 \\ \mathrm{H} & 3.57489 & 3.18436 & 3.26052 \\ \mathrm{H} & 1.55460 & 2.65088 & 4.75709 \\ \mathrm{C} & 1.23260 & 0.69808 & 3.83401 \\ \mathrm{H} & 1.96408 & 0.12699 & 4.42069 \\ \mathrm{H} & -1.30242 & -0.22334 & 6.06874 \\ \mathrm{C} & 1.23174 & 0.18518 & 2.37350 \\ \mathrm{H} & 1.66103 & -0.80955 & 2.26812 \\ \mathrm{H} & 0.23291 & 0.16790 & 1.94346 \\ \mathrm{H} & 2.08635 & 1.09147 & 1.50096 \\ \mathrm{H} & -0.11679 & 0.51459 & 4.48057 \\ \mathrm{H} & -0.97331 & 0.93148 & 3.95064 \\ \mathrm{H} & & -0.57109 & 6.19087\end{array}$




$\begin{array}{llll}\mathrm{C} & 0.43427 & 2.64049 & 0.14014 \\ \mathrm{~N} & -0.41420 & 1.47965 & -0.03427 \\ \mathrm{H} & 0.00288 & 0.65685 & -0.46345 \\ \mathrm{C} & -1.79015 & 1.51875 & 0.08805 \\ \mathrm{~S} & -2.60901 & 2.87498 & 0.67382 \\ \mathrm{~N} & -2.37017 & 0.34508 & -0.27692 \\ \mathrm{H} & -1.74391 & -0.46803 & -0.39645 \\ \mathrm{C} & -3.73765 & -0.00554 & -0.21196 \\ \mathrm{C} & -4.05151 & -1.27925 & 0.27981 \\ \mathrm{C} & -4.75750 & 0.83060 & -0.68086 \\ \mathrm{C} & -5.37934 & -1.70599 & 0.30299 \\ \mathrm{H} & -3.25476 & -1.90630 & 0.66660 \\ \mathrm{C} & -6.08098 & 0.39191 & -0.63657 \\ \mathrm{C} & -4.52080 & 1.81376 & -1.06355 \\ \mathrm{H} & -5.67112 & -3.98983 & -0.23795 \\ \mathrm{C} & -6.40430 & -0.87460 & -0.14820 \\ \mathrm{H} & -7.43644 & -1.20125 & -0.10747 \\ \mathrm{C} & -7.16901 & 1.27201 & -1.19329 \\ \mathrm{C} & -5.70474 & -3.09675 & 0.77951 \\ \mathrm{C} & -7.36468 & 1.04375 & -2.51453 \\ \mathrm{C} & -6.87824 & 2.58285 & -1.06226 \\ \mathrm{C} & -8.35472 & 1.05333 & -0.58132 \\ \mathrm{H} & -3.53162 & -3.53174 & 1.71184\end{array}$




$\begin{array}{llll}\mathrm{C} & -6.94365 & -3.16632 & 1.31787 \\ \mathrm{H} & -0.25359 & 3.42125 & 0.49336 \\ \mathrm{C} & 1.05319 & 3.15969 & -1.16239 \\ \mathrm{C} & 1.70088 & 4.44390 & -1.20326 \\ \mathrm{C} & 0.96518 & 2.46344 & -2.34573 \\ \mathrm{C} & 2.29197 & 4.84778 & -2.44838 \\ \mathrm{C} & 1.76413 & 5.34132 & -0.10993 \\ \mathrm{C} & 1.56568 & 2.96879 & -3.52562 \\ \mathrm{H} & 0.43729 & 1.52046 & -2.39166 \\ \mathrm{C} & 2.95690 & 6.09731 & -2.51928 \\ \mathrm{C} & 2.41009 & 6.56365 & -0.21482 \\ \mathrm{H} & 1.27222 & 5.12561 & 0.83100 \\ \mathrm{H} & 1.49434 & 2.39349 & -4.44853 \\ \mathrm{H} & 3.02694 & 6.94207 & -1.43417 \\ \mathrm{H} & 4.05849 & 8.58849 & 0.63458 \\ \mathrm{H} & 3.39911 & 6.37054 & -3.47206 \\ \mathrm{H} & 3.53732 & 7.89393 & -1.52504 \\ \mathrm{~N} & 2.22988 & 4.10105 & -3.58926 \\ \mathrm{H} & 2.40164 & 7.34074 & 0.90565 \\ \mathrm{H} & 2.98193 & 8.63583 & 0.84475 \\ \mathrm{H} & 2.49199 & 9.26305 & 0.08923 \\ \mathrm{H} & 2.82857 & 9.07603 & 1.83157 \\ \mathrm{H} & & -1.86997 & -0.71752\end{array}$




$\begin{array}{llll}\mathrm{O} & -0.92634 & -1.84571 & 1.46215 \\ \mathrm{~N} & -0.25136 & -2.24320 & 0.46952 \\ \mathrm{C} & 0.81303 & -3.05763 & 0.66825 \\ \mathrm{H} & 0.97948 & -3.29583 & 1.70650 \\ \mathrm{C} & 1.55395 & -3.64591 & -0.39951 \\ \mathrm{H} & 0.99756 & -3.71105 & -1.33220 \\ \mathrm{C} & 2.27007 & -4.91297 & -0.05361 \\ \mathrm{C} & 2.24331 & -5.99434 & -0.94957 \\ \mathrm{C} & 2.93788 & -5.07815 & 1.17334 \\ \mathrm{C} & 2.85111 & -7.20791 & -0.62320 \\ \mathrm{H} & 1.75436 & -5.88136 & -1.91259 \\ \mathrm{C} & 3.54575 & -6.28910 & 1.49708 \\ \mathrm{H} & 2.97973 & -4.25353 & 1.87894 \\ \mathrm{C} & 3.50211 & -7.36099 & 0.60113 \\ \mathrm{C} & 2.98407 & -2.43882 & -1.16570 \\ \mathrm{H} & 2.81354 & -8.03222 & -1.32988 \\ \mathrm{H} & 4.05050 & -6.39917 & 2.45337 \\ \mathrm{H} & 3.97294 & -8.30632 & 0.85667 \\ \mathrm{C} & 2.22620 & 0.57569 & 0.60303 \\ \mathrm{C} & 1.98521 & -0.26596 & -0.89852 \\ \mathrm{C} & 2.18631 & -1.27126 & -1.59977 \\ \mathrm{H} & 1.49882 & -1.33509 & -2.96224 \\ \mathrm{H} & & -2.06512 & -4.07390\end{array}$




$\begin{array}{llll}\mathrm{H} & 0.53869 & -1.83551 & -2.78295 \\ \mathrm{C} & 3.63868 & -3.25865 & -2.29301 \\ \mathrm{C} & 2.78264 & -3.38810 & -3.54233 \\ \mathrm{H} & 1.64386 & -2.23549 & -4.95064 \\ \mathrm{H} & 4.57522 & -2.76660 & -2.59583 \\ \mathrm{C} & 3.86883 & -2.20447 & 0.00507 \\ \mathrm{O} & 3.58856 & -1.56054 & 1.00815 \\ \mathrm{O} & 5.05976 & -2.83837 & -0.12105 \\ \mathrm{C} & 5.95269 & -2.72995 & 0.99372 \\ \mathrm{H} & 5.49971 & -3.15432 & 1.89370 \\ \mathrm{H} & 6.83997 & -3.29750 & 0.71188 \\ \mathrm{H} & 6.21221 & -1.68429 & 1.18232 \\ \mathrm{H} & 1.26811 & -0.31014 & -3.26088 \\ \mathrm{H} & 3.13752 & -1.45928 & -4.38467 \\ \mathrm{H} & 3.91092 & -4.25481 & -1.94382 \\ \mathrm{O} & 2.52811 & -4.45953 & -4.05968\end{array}$

TSI-3 (S,R)
$\begin{array}{llll}\mathrm{C} & 0.36723 & 2.98946 & 1.15203\end{array}$
$\begin{array}{llll}\text { C } & 0.82490 & 3.21297 & 3.62152\end{array}$
$\begin{array}{llll}\text { C } & -0.19815 & 3.48829 & 2.50085\end{array}$
$\begin{array}{llll}\mathrm{H} & 0.97881 & 3.77629 & 0.70763\end{array}$ 


$\begin{array}{llll}\mathrm{H} & -0.40307 & 4.56109 & 2.41565 \\ \mathrm{H} & -1.15671 & 3.00151 & 2.71073 \\ \mathrm{C} & 2.63468 & 2.59720 & 1.99777 \\ \mathrm{H} & 3.30083 & 1.80601 & 2.34010 \\ \mathrm{H} & 3.09293 & 3.07982 & 1.13195 \\ \mathrm{C} & 2.23191 & 3.59048 & 3.11140 \\ \mathrm{H} & 2.96276 & 3.53975 & 3.92528 \\ \mathrm{H} & 2.23598 & 4.62118 & 2.73879 \\ \mathrm{H} & 0.57500 & 3.78434 & 4.52031 \\ \mathrm{C} & 0.83590 & 1.69323 & 3.94065 \\ \mathrm{H} & 1.74907 & 1.47386 & 4.50846 \\ \mathrm{C} & 0.89010 & 0.95103 & 2.58238 \\ \mathrm{H} & 1.57872 & 0.10913 & 2.57800 \\ \mathrm{H} & -0.09190 & 0.60311 & 2.27060 \\ \mathrm{H} & -1.20383 & 1.27654 & 0.22035 \\ \mathrm{~N} & 1.38582 & 1.89712 & 1.49858 \\ \mathrm{C} & -0.33929 & 1.23080 & 4.76295 \\ \mathrm{H} & -1.32620 & 1.35611 & 4.31593 \\ \mathrm{H} & -0.23956 & 0.68466 & 5.97547 \\ \mathrm{H} & -1.11770 & 0.37129 & 6.53286 \\ \mathrm{H} & 0.72391 & 0.52694 & 6.45653 \\ \mathrm{H} & -0.73464 & 2.63744 & 0.10491 \\ \mathrm{H} & 0.51335 & 0.08352\end{array}$




\begin{tabular}{|c|c|c|c|}
\hline $\mathrm{C}$ & -2.53168 & 0.91838 & 0.19429 \\
\hline S & -3.77310 & 1.99010 & 0.57883 \\
\hline $\mathrm{N}$ & -2.66435 & -0.39348 & -0.17677 \\
\hline $\mathrm{H}$ & -1.82568 & -0.80007 & -0.60668 \\
\hline $\mathrm{C}$ & -3.76404 & -1.26474 & -0.17927 \\
\hline $\mathrm{C}$ & -3.59398 & -2.45955 & -0.90360 \\
\hline $\mathrm{C}$ & -4.96561 & -1.05150 & 0.50980 \\
\hline $\mathrm{C}$ & -4.60867 & -3.40933 & -0.94378 \\
\hline $\mathrm{H}$ & -2.66320 & -2.63402 & -1.43485 \\
\hline $\mathrm{C}$ & -5.97261 & -2.01790 & 0.45297 \\
\hline $\mathrm{H}$ & -5.11195 & -0.14396 & 1.07729 \\
\hline $\mathrm{C}$ & -5.81328 & -3.19803 & -0.26917 \\
\hline $\mathrm{H}$ & -6.60479 & -3.93637 & -0.30588 \\
\hline $\mathrm{C}$ & -7.27858 & -1.74143 & 1.15285 \\
\hline $\mathrm{C}$ & -4.39014 & -4.70962 & -1.67103 \\
\hline $\mathrm{C}$ & -8.09803 & -0.97736 & 0.39622 \\
\hline $\mathrm{C}$ & -7.09431 & -1.08377 & 2.31851 \\
\hline $\mathrm{C}$ & -7.95021 & -2.88133 & 1.43283 \\
\hline $\mathrm{C}$ & -3.45967 & -4.59972 & -2.64323 \\
\hline $\mathrm{C}$ & -3.96894 & -5.68393 & -0.83039 \\
\hline $\mathrm{C}$ & -5.52882 & -5.15716 & -2.24562 \\
\hline $\mathrm{H}$ & -1.59610 & 3.25975 & 0.37985 \\
\hline $\mathrm{C}$ & -0.34154 & 3.02606 & -1.32574 \\
\hline
\end{tabular}




$\begin{array}{llll}\mathrm{C} & -0.16703 & 4.40911 & -1.67903 \\ \mathrm{C} & -0.19370 & 2.08858 & -2.32229 \\ \mathrm{C} & 0.21345 & 4.70230 & -3.03159 \\ \mathrm{C} & -0.36456 & 5.49103 & -0.78753 \\ \mathrm{C} & 0.15528 & 2.49836 & -3.63513 \\ \mathrm{H} & -0.34246 & 1.03147 & -2.12836 \\ \mathrm{C} & 0.41327 & 6.05276 & -3.41085 \\ \mathrm{C} & -0.16870 & 6.80302 & -1.19094 \\ \mathrm{H} & -0.70963 & 5.33589 & 0.22813 \\ \mathrm{H} & 0.25783 & 1.74512 & -4.41590 \\ \mathrm{C} & 0.23530 & 7.08776 & -2.51976 \\ \mathrm{H} & 0.70329 & 6.24237 & -4.43946 \\ \mathrm{H} & 0.39171 & 8.11009 & -2.84398 \\ \mathrm{~N} & 0.37415 & 3.74451 & -3.99129 \\ \mathrm{O} & -0.38857 & 7.75803 & -0.24214 \\ \mathrm{H} & 0.65780 & -1.52937 & -0.76355 \\ \mathrm{H} & -0.26608 & 9.12519 & -0.60639 \\ \mathrm{H} & -0.96603 & 9.39461 & -1.40761 \\ \mathrm{H} & -0.51186 & 9.69462 & 0.29169 \\ \mathrm{H} & 0.75681 & 9.37118 & -0.92055 \\ \mathrm{H} & -0.25455 & -1.38534 & -1.64591 \\ \mathrm{H} & 0.55173 & -0.91422 & 0.35011 \\ \mathrm{H} & & -2.36209 & -1.00954\end{array}$




$\begin{array}{llll}\mathrm{H} & 1.64182 & -2.85881 & -1.96584 \\ \mathrm{C} & 2.71494 & -2.57265 & -0.04367 \\ \mathrm{H} & 2.48027 & -2.15379 & 0.92910 \\ \mathrm{C} & 3.40671 & -3.88000 & -0.02561 \\ \mathrm{C} & 3.72307 & -4.46172 & 1.21569 \\ \mathrm{C} & 3.70837 & -4.59971 & -1.19582 \\ \mathrm{C} & 4.32149 & -5.71868 & 1.28614 \\ \mathrm{H} & 3.47521 & -3.92657 & 2.12951 \\ \mathrm{C} & 4.31962 & -5.84946 & -1.12538 \\ \mathrm{H} & 3.47052 & -4.17836 & -2.16808 \\ \mathrm{C} & 4.62840 & -6.41391 & 0.11426 \\ \mathrm{H} & 4.54543 & -6.15652 & 2.25512 \\ \mathrm{H} & 4.55600 & -6.38415 & -2.04075 \\ \mathrm{H} & 5.10233 & -7.38988 & 0.16640 \\ \mathrm{H} & 3.75120 & -0.29503 & 1.89950 \\ \mathrm{H} & 1.68520 & 1.32737 & 0.68112 \\ \mathrm{O} & 2.83248 & 0.72972 & -0.65639 \\ \mathrm{C} & 3.57370 & -0.16540 & -1.05628 \\ \mathrm{C} & 4.35179 & -1.13563 & -0.26005 \\ \mathrm{C} & 5.52108 & -1.77410 & -1.01484 \\ \mathrm{H} & 6.15606 & -0.98282 & -1.44982 \\ \mathrm{H} & 4.60710 & -0.76728 & 1.14387 \\ \mathrm{H} & & -0.99660 & 1.67131\end{array}$




$\begin{array}{llll}\mathrm{O} & 3.74732 & -0.38335 & -2.37919 \\ \mathrm{C} & 2.95468 & 0.41157 & -3.27387 \\ \mathrm{H} & 3.06233 & 1.47542 & -3.05394 \\ \mathrm{H} & 3.32989 & 0.18428 & -4.27243 \\ \mathrm{H} & 1.90294 & 0.12832 & -3.18874 \\ \mathrm{C} & 6.59418 & -2.36587 & 1.28365 \\ \mathrm{C} & 6.47828 & -2.64890 & -0.20836 \\ \mathrm{H} & 5.18219 & -2.35914 & -1.87165 \\ \mathrm{H} & 6.66208 & -0.20361 & 1.25284 \\ \mathrm{H} & 6.00475 & -0.85802 & 2.75597 \\ \mathrm{H} & 7.64488 & -2.46858 & 1.57510 \\ \mathrm{H} & 6.04918 & -3.16481 & 1.80174 \\ \mathrm{O} & 7.15387 & -3.50201 & -0.74903\end{array}$

TSI-4 (R,R)
$\begin{array}{llll}\text { C } & 1.28888 & -2.65415 & 1.26956\end{array}$
$\begin{array}{llll}\text { C } & 0.69622 & -2.80912 & 3.70991\end{array}$
$\begin{array}{llll}\text { C } & 1.82076 & -2.47450 & 2.70801\end{array}$
$\mathrm{H} \quad 1.40371 \quad-3.70212 \quad 0.98441$
$\begin{array}{llll}\mathrm{H} & 2.67847 & -3.14173 & 2.84305\end{array}$
$\mathrm{H} \quad 2.18791 \quad-1.45336 \quad 2.85535$
$\begin{array}{llll}\text { C } & -0.80662 & -3.74052 & 1.92021\end{array}$ 


\begin{tabular}{|c|c|c|c|}
\hline $\mathrm{H}$ & -1.87083 & -3.55643 & 2.05986 \\
\hline $\mathrm{H}$ & -0.69315 & -4.51419 & 1.15661 \\
\hline $\mathrm{C}$ & -0.04930 & -4.07380 & 3.22875 \\
\hline $\mathrm{H}$ & -0.76537 & -4.40731 & 3.98696 \\
\hline $\mathrm{H}$ & 0.65803 & -4.89595 & 3.07124 \\
\hline $\mathrm{H}$ & 1.10577 & -2.96439 & 4.71205 \\
\hline $\mathrm{C}$ & -0.32866 & -1.64249 & 3.72704 \\
\hline $\mathrm{H}$ & -1.24411 & -2.01015 & 4.20883 \\
\hline $\mathrm{C}$ & -0.62851 & -1.29793 & 2.24943 \\
\hline $\mathrm{H}$ & -1.68502 & -1.13193 & 2.05179 \\
\hline $\mathrm{H}$ & -0.07806 & -0.42388 & 1.90921 \\
\hline $\mathrm{N}$ & -0.22900 & -2.46232 & 1.35136 \\
\hline $\mathrm{C}$ & 0.13294 & -0.42705 & 4.49032 \\
\hline $\mathrm{H}$ & 0.96769 & 0.13008 & 4.06525 \\
\hline $\mathrm{C}$ & -0.41934 & -0.00683 & 5.62865 \\
\hline $\mathrm{H}$ & -0.04958 & 0.87140 & 6.14968 \\
\hline $\mathrm{H}$ & -1.26857 & -0.51803 & 6.07864 \\
\hline $\mathrm{C}$ & 2.03829 & -1.81921 & 0.18118 \\
\hline $\mathrm{N}$ & 1.45721 & -0.51311 & -0.05789 \\
\hline $\mathrm{H}$ & 0.55707 & -0.47065 & -0.53155 \\
\hline $\mathrm{C}$ & 2.16648 & 0.66251 & 0.08761 \\
\hline $\mathrm{S}$ & 3.74826 & 0.71086 & 0.67265 \\
\hline $\mathrm{N}$ & 1.43142 & 1.75609 & -0.26230 \\
\hline
\end{tabular}




$\begin{array}{llll}\mathrm{H} & 0.40538 & 1.65394 & -0.30955 \\ \mathrm{C} & 1.84482 & 3.10887 & -0.21013 \\ \mathrm{C} & 0.98057 & 4.03228 & 0.38853 \\ \mathrm{C} & 3.03534 & 3.54781 & -0.80108 \\ \mathrm{C} & 1.31468 & 5.38796 & 0.39739 \\ \mathrm{H} & 0.07062 & 3.67543 & 0.85988 \\ \mathrm{C} & 3.35855 & 4.90339 & -0.77223 \\ \mathrm{H} & 3.70324 & 2.83710 & -1.26869 \\ \mathrm{C} & 2.50439 & 5.83345 & -0.17591 \\ \mathrm{H} & 2.76922 & 6.88355 & -0.14838 \\ \mathrm{C} & 4.60797 & 5.38338 & -1.46211 \\ \mathrm{C} & 0.35416 & 6.37939 & 0.99894 \\ \mathrm{C} & 4.35797 & 5.74147 & -2.74522 \\ \mathrm{C} & 5.56485 & 4.43320 & -1.50060 \\ \mathrm{C} & 5.13376 & 6.46929 & -0.85191 \\ \mathrm{C} & -0.59091 & 6.75948 & 0.10679 \\ \mathrm{C} & -0.29764 & 5.86785 & 2.06452 \\ \mathrm{C} & 0.98165 & 7.50531 & 1.40765 \\ \mathrm{C} & 3.01894 & -1.58923 & 0.61273 \\ \mathrm{C} & 2.28475 & -2.66227 & -1.07299 \\ \mathrm{C} & 3.30562 & -3.67552 & -1.05738 \\ \mathrm{C} & 1.54886 & -2.51937 & -2.22603 \\ \mathrm{C} & 3.46309 & -4.47211 & -2.24211\end{array}$




\begin{tabular}{|c|c|c|c|}
\hline $\mathrm{C}$ & 4.15747 & -3.93043 & 0.04357 \\
\hline $\mathrm{C}$ & 1.79602 & -3.35887 & -3.34174 \\
\hline $\mathrm{H}$ & 0.75873 & -1.78372 & -2.28679 \\
\hline $\mathrm{C}$ & 4.45485 & -5.48393 & -2.26110 \\
\hline $\mathrm{C}$ & 5.11768 & -4.92943 & -0.00570 \\
\hline $\mathrm{H}$ & 4.11253 & -3.33593 & 0.94825 \\
\hline $\mathrm{H}$ & 1.19964 & -3.23422 & -4.24558 \\
\hline $\mathrm{C}$ & 5.26656 & -5.71952 & -1.17394 \\
\hline $\mathrm{H}$ & 4.55241 & -6.06747 & -3.17102 \\
\hline $\mathrm{H}$ & 6.01697 & -6.50016 & -1.22158 \\
\hline $\mathrm{N}$ & 2.70652 & -4.30621 & -3.36644 \\
\hline $\mathrm{O}$ & 5.87574 & -5.08317 & 1.11646 \\
\hline $\mathrm{C}$ & 6.92496 & -6.04044 & 1.11030 \\
\hline $\mathrm{H}$ & 7.66427 & -5.82479 & 0.32844 \\
\hline $\mathrm{H}$ & 7.40324 & -5.96171 & 2.08810 \\
\hline $\mathrm{H}$ & 6.54252 & -7.06097 & 0.97720 \\
\hline $\mathrm{O}$ & -1.44279 & 1.66762 & -0.58126 \\
\hline $\mathrm{O}$ & -1.17492 & 1.65925 & 1.60685 \\
\hline $\mathrm{N}$ & -1.88602 & 1.41081 & 0.59231 \\
\hline $\mathrm{C}$ & -3.12480 & 0.87301 & 0.76002 \\
\hline $\mathrm{H}$ & -3.39139 & 0.73074 & 1.79509 \\
\hline $\mathrm{C}$ & -4.01331 & 0.64210 & -0.32285 \\
\hline $\mathrm{H}$ & -3.79476 & 1.22804 & -1.21293 \\
\hline
\end{tabular}




\begin{tabular}{|c|c|c|c|}
\hline $\mathrm{C}$ & -5.46145 & 0.60074 & 0.03675 \\
\hline $\mathrm{C}$ & -6.40095 & 1.23980 & -0.78746 \\
\hline $\mathrm{C}$ & -5.91954 & -0.01871 & 1.21455 \\
\hline $\mathrm{C}$ & -7.75585 & 1.25897 & -0.45215 \\
\hline $\mathrm{H}$ & -6.06599 & 1.73508 & -1.69475 \\
\hline $\mathrm{C}$ & -7.27131 & 0.00435 & 1.55217 \\
\hline $\mathrm{H}$ & -5.21539 & -0.53360 & 1.86115 \\
\hline $\mathrm{C}$ & -8.19628 & 0.64207 & 0.71879 \\
\hline $\mathrm{H}$ & -8.46415 & 1.75686 & -1.10796 \\
\hline $\mathrm{H}$ & -7.60515 & -0.47678 & 2.46778 \\
\hline $\mathrm{H}$ & -9.24994 & 0.65811 & 0.98298 \\
\hline $\mathrm{H}$ & -0.66863 & -2.26705 & 0.43173 \\
\hline $\mathrm{O}$ & -1.22777 & -1.23763 & -1.13224 \\
\hline $\mathrm{C}$ & -2.24232 & -0.85486 & -1.72654 \\
\hline $\mathrm{C}$ & -3.65403 & -1.10103 & -1.34211 \\
\hline $\mathrm{C}$ & -4.60766 & -1.08901 & -2.54556 \\
\hline $\mathrm{H}$ & -4.17530 & -1.68095 & -3.36787 \\
\hline $\mathrm{C}$ & -3.85428 & -2.17393 & -0.35179 \\
\hline $\mathrm{O}$ & -3.12774 & -2.31651 & 0.63759 \\
\hline $\mathrm{O}$ & -2.18260 & -0.15817 & -2.87413 \\
\hline $\mathrm{C}$ & -0.90542 & 0.27462 & -3.35890 \\
\hline $\mathrm{H}$ & -1.12753 & 0.94813 & -4.18716 \\
\hline $\mathrm{H}$ & -0.36142 & 0.80653 & -2.57800 \\
\hline
\end{tabular}




$\begin{array}{llll}\mathrm{H} & -0.32341 & -0.57617 & -3.72344 \\ \mathrm{H} & -4.70962 & -0.08361 & -2.96382 \\ \mathrm{C} & -4.99267 & -3.16464 & -0.58644 \\ \mathrm{H} & -4.59656 & -3.92261 & -1.28147 \\ \mathrm{H} & -5.18610 & -3.68018 & 0.35896 \\ \mathrm{C} & -6.27485 & -2.56372 & -1.17444 \\ \mathrm{C} & -6.01741 & -1.64986 & -2.36067 \\ \mathrm{O} & -6.88633 & -1.39752 & -3.17251 \\ \mathrm{H} & -6.97858 & -3.34184 & -1.48834 \\ \mathrm{H} & -6.79277 & -1.96348 & -0.41671\end{array}$

TSI-5 (R,R)

$\begin{array}{llll}\mathrm{C} & 3.60608 & -0.79981 & 1.17144 \\ \mathrm{C} & 3.79758 & -0.52762 & 3.67055 \\ \mathrm{C} & 4.13035 & 0.11415 & 2.30603 \\ \mathrm{H} & 4.38612 & -1.51794 & 0.90885 \\ \mathrm{H} & 5.21272 & 0.23391 & 2.19089 \\ \mathrm{H} & 3.68899 & 1.11261 & 2.22537 \\ \mathrm{C} & 3.14973 & -2.71776 & 2.62595 \\ \mathrm{H} & 2.33442 & -3.25117 & 3.11946 \\ \mathrm{H} & 3.64790 & -3.41657 & 1.94938 \\ \mathrm{C} & 4.12629 & -2.03646 & 3.61614\end{array}$




$\begin{array}{llll}\mathrm{H} & 4.02504 & -2.48978 & 4.60776 \\ \mathrm{H} & 5.16451 & -2.18615 & 3.29864 \\ \mathrm{H} & 4.36527 & -0.04204 & 4.46970 \\ \mathrm{C} & 2.27390 & -0.39285 & 3.94297 \\ \mathrm{H} & 2.02049 & -1.07570 & 4.76470 \\ \mathrm{C} & 1.55602 & -0.85073 & 2.64930 \\ \mathrm{H} & 0.69352 & -1.49134 & 2.82655 \\ \mathrm{H} & 1.21681 & -0.00622 & 2.05524 \\ \mathrm{~N} & 2.50438 & -1.65345 & 1.78189 \\ \mathrm{C} & 1.83646 & 0.99252 & 4.34481 \\ \mathrm{H} & 1.94636 & 1.77500 & 3.59302 \\ \mathrm{C} & 1.32487 & 1.29936 & 5.53781 \\ \mathrm{H} & 1.02759 & 2.31439 & 5.78540 \\ \mathrm{H} & 1.18317 & 0.54838 & 6.31316 \\ \mathrm{C} & -0.93581 & 2.65460 & -0.37479 \\ \mathrm{C} & 3.20464 & -0.06202 & -0.16153 \\ \mathrm{~N} & 1.78789 & 0.23627 & -0.26324 \\ \mathrm{H} & 1.16014 & -0.55098 & -0.42098 \\ \mathrm{C} & 1.26404 & 1.50333 & -0.19739 \\ \mathrm{H} & 2.20562 & 2.86784 & 0.14637 \\ \mathrm{H} & -0.08366 & 1.52677 & -0.38737 \\ \mathrm{H} & -2.14654 & 2.54223 & 0.31635\end{array}$




$\begin{array}{llll}\mathrm{C} & -0.64656 & 3.83136 & -1.07738 \\ \mathrm{C} & -3.06095 & 3.59644 & 0.30224 \\ \mathrm{H} & -2.34844 & 1.63846 & 0.87850 \\ \mathrm{C} & -1.56162 & 4.88313 & -1.06449 \\ \mathrm{H} & 0.28446 & 3.92514 & -1.61885 \\ \mathrm{C} & -2.77488 & 4.77688 & -0.38063 \\ \mathrm{H} & -3.47522 & 5.60368 & -0.37069 \\ \mathrm{C} & -1.27800 & 6.13287 & -1.85699 \\ \mathrm{C} & -4.38425 & 3.42945 & 0.99672 \\ \mathrm{C} & -1.87561 & 6.09478 & -3.07182 \\ \mathrm{C} & 0.04050 & 6.31309 & -2.07085 \\ \mathrm{C} & -1.74644 & 7.23546 & -1.22922 \\ \mathrm{C} & -5.30131 & 2.83599 & 0.18488 \\ \mathrm{C} & -4.28685 & 2.64990 & 2.09621 \\ \mathrm{C} & -4.91412 & 4.61009 & 1.37640 \\ \mathrm{H} & 3.68284 & 0.92174 & -0.11128 \\ \mathrm{C} & 3.76223 & -0.78740 & -1.38934 \\ \mathrm{C} & 5.17733 & -0.75504 & -1.64488 \\ \mathrm{C} & 2.97219 & -1.47999 & -2.27865 \\ \mathrm{C} & 5.66143 & -1.48298 & -2.78360 \\ \mathrm{H} & 6.11178 & -0.04538 & -0.85334 \\ \mathrm{H} & 3.55981 & -2.15616 & -3.37844 \\ \mathrm{H} & & -1.54962 & -2.14464\end{array}$




\begin{tabular}{|c|c|c|c|}
\hline $\mathrm{C}$ & 7.05139 & -1.48076 & -3.05826 \\
\hline $\mathrm{C}$ & 7.46589 & -0.06027 & -1.15019 \\
\hline $\mathrm{H}$ & 5.79947 & 0.55880 & -0.00996 \\
\hline $\mathrm{H}$ & 2.91837 & -2.70768 & -4.06500 \\
\hline $\mathrm{C}$ & 7.94376 & -0.79345 & -2.26572 \\
\hline $\mathrm{H}$ & 7.38711 & -2.04066 & -3.92538 \\
\hline $\mathrm{H}$ & 9.00066 & -0.81077 & -2.50557 \\
\hline $\mathrm{N}$ & 4.84901 & -2.17787 & -3.63255 \\
\hline $\mathrm{O}$ & 8.26798 & 0.65987 & -0.31430 \\
\hline $\mathrm{C}$ & 9.65534 & 0.74617 & -0.60378 \\
\hline $\mathrm{H}$ & 9.83548 & 1.19210 & -1.59031 \\
\hline $\mathrm{H}$ & 10.07925 & 1.39363 & 0.16585 \\
\hline $\mathrm{H}$ & 10.14234 & -0.23676 & -0.55584 \\
\hline $\mathrm{H}$ & 1.91186 & -2.12993 & 1.02035 \\
\hline $\mathrm{O}$ & -2.00532 & -0.44182 & -1.22325 \\
\hline $\mathrm{C}$ & -3.14209 & -0.82104 & -0.90598 \\
\hline $\mathrm{C}$ & -4.26863 & -0.62642 & -1.91979 \\
\hline $\mathrm{C}$ & -3.44092 & -1.52014 & 0.34857 \\
\hline $\mathrm{C}$ & -5.64777 & -0.41172 & -1.27706 \\
\hline $\mathrm{H}$ & -3.98447 & 0.21663 & -2.55626 \\
\hline $\mathrm{C}$ & -4.89114 & -1.84637 & 0.71100 \\
\hline $\mathrm{C}$ & -5.94988 & -1.57085 & -0.35158 \\
\hline $\mathrm{H}$ & -5.63299 & 0.51698 & -0.68941 \\
\hline
\end{tabular}




\begin{tabular}{|c|c|c|c|}
\hline $\mathrm{H}$ & -5.01950 & -2.88071 & 1.04282 \\
\hline $\mathrm{C}$ & -2.56524 & -1.22076 & 1.50333 \\
\hline $\mathrm{O}$ & -1.52386 & -0.57667 & 1.50031 \\
\hline $\mathrm{O}$ & -3.06356 & -1.77266 & 2.64335 \\
\hline $\mathrm{C}$ & -2.26746 & -1.61693 & 3.82246 \\
\hline $\mathrm{H}$ & -1.97465 & -0.57361 & 3.96433 \\
\hline $\mathrm{H}$ & -2.89600 & -1.95522 & 4.64781 \\
\hline $\mathrm{H}$ & -1.37295 & -2.24214 & 3.74741 \\
\hline $\mathrm{H}$ & -4.30182 & -1.51555 & -2.56459 \\
\hline $\mathrm{H}$ & -6.44050 & -0.32935 & -2.02569 \\
\hline $\mathrm{H}$ & -5.18763 & -1.23402 & 1.57646 \\
\hline $\mathrm{O}$ & -6.97380 & -2.22391 & -0.41018 \\
\hline $\mathrm{O}$ & 0.99365 & -2.60771 & -0.16085 \\
\hline $\mathrm{N}$ & -0.14291 & -3.03530 & 0.32028 \\
\hline $\mathrm{O}$ & -0.21907 & -3.34931 & 1.53656 \\
\hline $\mathrm{C}$ & -1.16446 & -3.15449 & -0.55565 \\
\hline $\mathrm{H}$ & -0.89654 & -2.90220 & -1.56820 \\
\hline $\mathrm{C}$ & -2.47563 & -3.45556 & -0.13147 \\
\hline $\mathrm{H}$ & -2.55876 & -3.76648 & 0.90540 \\
\hline $\mathrm{C}$ & -3.42536 & -4.11531 & -1.05127 \\
\hline $\mathrm{C}$ & -3.34306 & -3.98880 & -2.45274 \\
\hline $\mathrm{C}$ & -4.42611 & -4.94894 & -0.51966 \\
\hline $\mathrm{C}$ & -4.24012 & -4.65663 & -3.28230 \\
\hline
\end{tabular}




$\begin{array}{llll}\mathrm{H} & -2.57385 & -3.36536 & -2.89814 \\ \mathrm{C} & -5.32637 & -5.61415 & -1.35085 \\ \mathrm{H} & -4.48621 & -5.08780 & 0.55628 \\ \mathrm{C} & -5.23898 & -5.46795 & -2.73514 \\ \mathrm{H} & -4.15900 & -4.54547 & -4.36015 \\ \mathrm{H} & -6.09397 & -6.24693 & -0.91502 \\ \mathrm{H} & -5.93956 & -5.98437 & -3.38514\end{array}$

TSI-6(S,R)

$\begin{array}{llll}\mathrm{C} & 2.30548 & -2.65842 & 0.99550 \\ \mathrm{C} & 3.74462 & -3.85890 & 2.69503 \\ \mathrm{C} & 3.63132 & -3.43591 & 1.21821 \\ \mathrm{H} & 1.54968 & -3.28510 & 0.52132 \\ \mathrm{H} & 3.65720 & -4.31714 & 0.56976 \\ \mathrm{H} & 4.48770 & -2.81590 & 0.93244 \\ \mathrm{C} & 1.26529 & -3.66680 & 2.98627 \\ \mathrm{H} & 0.86418 & -3.39972 & 3.96692 \\ \mathrm{H} & 0.44062 & -4.03590 & 2.37540 \\ \mathrm{C} & 2.46849 & -4.63187 & 3.07726 \\ \mathrm{H} & 2.55204 & -5.03689 & 4.09144 \\ \mathrm{H} & 2.33079 & -5.48314 & 2.40113 \\ \mathrm{H} & 4.62938 & -4.48703 & 2.83684\end{array}$




\begin{tabular}{|c|c|c|c|}
\hline $\mathrm{C}$ & 3.84990 & -2.60953 & 3.61833 \\
\hline $\mathrm{H}$ & 3.73192 & -2.96329 & 4.65059 \\
\hline $\mathrm{C}$ & 2.66241 & -1.66258 & 3.27040 \\
\hline $\mathrm{H}$ & 2.09385 & -1.37129 & 4.15700 \\
\hline $\mathrm{H}$ & 2.98034 & -0.74208 & 2.77480 \\
\hline $\mathrm{N}$ & 1.70459 & -2.36838 & 2.35330 \\
\hline $\mathrm{C}$ & 5.17436 & -1.89459 & 3.53165 \\
\hline $\mathrm{H}$ & 5.40001 & -1.39490 & 2.58916 \\
\hline $\mathrm{C}$ & 6.06124 & -1.83341 & 4.52565 \\
\hline $\mathrm{H}$ & 7.00626 & -1.30937 & 4.41499 \\
\hline $\mathrm{H}$ & 5.87463 & -2.30060 & 5.49087 \\
\hline $\mathrm{C}$ & 2.52638 & -1.38474 & 0.13639 \\
\hline $\mathrm{N}$ & 1.36738 & -0.50355 & 0.07690 \\
\hline $\mathrm{H}$ & 0.57227 & -0.80639 & -0.49247 \\
\hline $\mathrm{C}$ & 1.38232 & 0.81066 & 0.46588 \\
\hline $\mathrm{S}$ & 2.66899 & 1.50225 & 1.32962 \\
\hline $\mathrm{N}$ & 0.24237 & 1.47464 & 0.10954 \\
\hline $\mathrm{H}$ & -0.51537 & 0.89357 & -0.25732 \\
\hline $\mathrm{C}$ & -0.08801 & 2.84206 & 0.22971 \\
\hline $\mathrm{C}$ & -1.45289 & 3.15505 & 0.29596 \\
\hline $\mathrm{C}$ & 0.85089 & 3.88379 & 0.19531 \\
\hline $\mathrm{C}$ & -1.86735 & 4.48589 & 0.33189 \\
\hline $\mathrm{H}$ & -2.18813 & 2.35862 & 0.30102 \\
\hline
\end{tabular}




\begin{tabular}{|c|c|c|c|}
\hline $\mathrm{C}$ & 0.41582 & 5.20790 & 0.25427 \\
\hline $\mathrm{H}$ & 1.90554 & 3.66591 & 0.11898 \\
\hline $\mathrm{C}$ & -0.94088 & 5.52698 & 0.32154 \\
\hline $\mathrm{H}$ & -1.26541 & 6.55968 & 0.35544 \\
\hline $\mathrm{C}$ & 1.43644 & 6.31501 & 0.29020 \\
\hline $\mathrm{C}$ & -3.34270 & 4.78078 & 0.31766 \\
\hline $\mathrm{C}$ & 0.96579 & 7.44936 & -0.27837 \\
\hline $\mathrm{C}$ & 2.57342 & 5.98013 & -0.35733 \\
\hline $\mathrm{C}$ & 1.78405 & 6.63118 & 1.55889 \\
\hline $\mathrm{C}$ & -3.86112 & 4.66023 & -0.93410 \\
\hline $\mathrm{C}$ & -4.04071 & 3.93233 & 1.10331 \\
\hline $\mathrm{C}$ & -3.61831 & 6.03649 & 0.73110 \\
\hline $\mathrm{H}$ & 3.31230 & -0.79645 & 0.60763 \\
\hline $\mathrm{C}$ & 2.98187 & -1.79643 & -1.27032 \\
\hline $\mathrm{C}$ & 4.12503 & -1.19802 & -1.90166 \\
\hline $\mathrm{C}$ & 2.28772 & -2.75597 & -1.97981 \\
\hline $\mathrm{C}$ & 4.47324 & -1.67891 & -3.21331 \\
\hline $\mathrm{C}$ & 4.92034 & -0.18226 & -1.32025 \\
\hline $\mathrm{C}$ & 2.71124 & -3.13862 & -3.27588 \\
\hline $\mathrm{H}$ & 1.40649 & -3.23443 & -1.56344 \\
\hline $\mathrm{C}$ & 5.60801 & -1.13431 & -3.86400 \\
\hline $\mathrm{C}$ & 6.02275 & 0.32976 & -1.98714 \\
\hline $\mathrm{H}$ & 4.66749 & 0.26161 & -0.36354 \\
\hline
\end{tabular}




\begin{tabular}{|c|c|c|c|}
\hline $\mathrm{H}$ & 2.15177 & -3.89910 & -3.82017 \\
\hline $\mathrm{C}$ & 6.37439 & -0.15568 & -3.27347 \\
\hline $\mathrm{H}$ & 5.84786 & -1.51832 & -4.85034 \\
\hline $\mathrm{H}$ & 7.23625 & 0.24140 & -3.79743 \\
\hline $\mathrm{N}$ & 3.76666 & -2.63751 & -3.87880 \\
\hline $\mathrm{O}$ & 6.71648 & 1.30123 & -1.33180 \\
\hline $\mathrm{C}$ & 7.81526 & 1.92064 & -1.98265 \\
\hline $\mathrm{H}$ & 7.50957 & 2.40946 & -2.91673 \\
\hline $\mathrm{H}$ & 8.18361 & 2.67604 & -1.28649 \\
\hline $\mathrm{H}$ & 8.61915 & 1.20320 & -2.19479 \\
\hline $\mathrm{H}$ & 0.78413 & -1.81628 & 2.23838 \\
\hline $\mathrm{O}$ & -1.21144 & -0.68356 & -1.21218 \\
\hline $\mathrm{C}$ & -2.06408 & -1.47344 & -1.65197 \\
\hline $\mathrm{C}$ & -1.55704 & -2.73981 & -2.35028 \\
\hline $\mathrm{C}$ & -3.50256 & -1.30471 & -1.45219 \\
\hline $\mathrm{C}$ & -2.51805 & -3.37179 & -3.37424 \\
\hline $\mathrm{H}$ & -0.59920 & -2.48734 & -2.81476 \\
\hline $\mathrm{C}$ & -4.45775 & -2.10665 & -2.33976 \\
\hline $\mathrm{C}$ & -3.89603 & -3.45516 & -2.75144 \\
\hline $\mathrm{H}$ & -2.57081 & -2.73843 & -4.27053 \\
\hline $\mathrm{H}$ & -5.42066 & -2.25452 & -1.84698 \\
\hline $\mathrm{C}$ & -3.96683 & 0.05430 & -1.08466 \\
\hline $\mathrm{O}$ & -3.40866 & 0.81951 & -0.31503 \\
\hline
\end{tabular}




\begin{tabular}{|c|c|c|c|}
\hline $\mathrm{O}$ & -5.14779 & 0.36633 & -1.67364 \\
\hline $\mathrm{C}$ & -5.68748 & 1.65327 & -1.33034 \\
\hline $\mathrm{H}$ & -4.98628 & 2.45175 & -1.58124 \\
\hline $\mathrm{H}$ & -6.60494 & 1.74520 & -1.91270 \\
\hline $\mathrm{H}$ & -5.90693 & 1.70395 & -0.26068 \\
\hline $\mathrm{H}$ & -1.34482 & -3.45919 & -1.54905 \\
\hline $\mathrm{H}$ & -2.18407 & -4.36884 & -3.67453 \\
\hline $\mathrm{H}$ & -4.66506 & -1.53832 & -3.25958 \\
\hline $\mathrm{O}$ & -4.48227 & -4.50612 & -2.56395 \\
\hline $\mathrm{C}$ & -2.73773 & -1.55967 & 1.38424 \\
\hline $\mathrm{C}$ & -3.64161 & -2.21312 & 0.51717 \\
\hline $\mathrm{C}$ & -5.09459 & -2.13854 & 0.80178 \\
\hline $\mathrm{C}$ & -5.91555 & -3.22898 & 0.46016 \\
\hline $\mathrm{C}$ & -5.68415 & -1.03211 & 1.44339 \\
\hline $\mathrm{C}$ & -7.27768 & -3.22095 & 0.76251 \\
\hline $\mathrm{H}$ & -5.48453 & -4.08157 & -0.05746 \\
\hline $\mathrm{C}$ & -7.04438 & -1.02634 & 1.74126 \\
\hline $\mathrm{H}$ & -5.07881 & -0.16942 & 1.70131 \\
\hline $\mathrm{C}$ & -7.84653 & -2.12162 & 1.40641 \\
\hline $\mathrm{H}$ & -7.89186 & -4.07551 & 0.49232 \\
\hline $\mathrm{H}$ & -7.48031 & -0.16509 & 2.24050 \\
\hline $\mathrm{H}$ & -8.90674 & -2.11483 & 1.64441 \\
\hline $\mathrm{H}$ & -3.30491 & -3.18318 & 0.16437 \\
\hline
\end{tabular}




$\begin{array}{llll}\mathrm{H} & -2.97974 & -0.69656 & 1.98144 \\ \mathrm{~N} & -1.45341 & -1.96302 & 1.51249 \\ \mathrm{O} & -0.98161 & -2.95638 & 0.87458 \\ \mathrm{O} & -0.71062 & -1.32352 & 2.35059\end{array}$

TSI-7 (R,S)

$\begin{array}{lrrr}\mathrm{C} & 3.58390 & -1.18114 & 0.93844 \\ \mathrm{C} & 3.80522 & -1.94578 & 3.32541 \\ \mathrm{C} & 4.03065 & -0.76304 & 2.35760 \\ \mathrm{H} & 4.42356 & -1.66878 & 0.43839 \\ \mathrm{H} & 5.08867 & -0.48274 & 2.32569 \\ \mathrm{H} & 3.47877 & 0.12386 & 2.68684 \\ \mathrm{C} & 3.31873 & -3.55721 & 1.45718 \\ \mathrm{H} & 2.56699 & -4.31484 & 1.68994 \\ \mathrm{H} & 3.85192 & -3.87298 & 0.55719 \\ \mathrm{C} & 4.26663 & -3.25532 & 2.64558 \\ \mathrm{H} & 4.24215 & -4.08693 & 3.35732 \\ \mathrm{H} & 5.30168 & -3.15919 & 2.29900 \\ \mathrm{H} & 4.35181 & -1.78816 & 4.25976 \\ \mathrm{C} & 2.28569 & -2.08536 & 3.61211 \\ \mathrm{H} & 2.11909 & -3.07317 & 4.06162 \\ \mathrm{C} & 1.57425 & -2.01767 & 2.23847 \\ & & & 48\end{array}$




\begin{tabular}{|c|c|c|c|}
\hline $\mathrm{H}$ & 0.78321 & -2.75809 & 2.12160 \\
\hline $\mathrm{H}$ & 1.13661 & -1.04133 & 2.04115 \\
\hline $\mathrm{N}$ & 2.56404 & -2.29922 & 1.12315 \\
\hline $\mathrm{C}$ & 1.73337 & -1.05332 & 4.56109 \\
\hline $\mathrm{H}$ & 1.78082 & -0.01434 & 4.23354 \\
\hline $\mathrm{C}$ & 1.19204 & -1.33818 & 5.74645 \\
\hline $\mathrm{H}$ & 0.80855 & -0.55928 & 6.39925 \\
\hline $\mathrm{H}$ & 1.11084 & -2.36093 & 6.11001 \\
\hline $\mathrm{C}$ & 3.13800 & -0.00626 & 0.00355 \\
\hline $\mathrm{N}$ & 1.71586 & 0.27410 & 0.06572 \\
\hline $\mathrm{H}$ & 1.09512 & -0.42132 & -0.34459 \\
\hline $\mathrm{C}$ & 1.20476 & 1.52414 & 0.33182 \\
\hline S & 2.16581 & 2.80155 & 0.87581 \\
\hline $\mathrm{N}$ & -0.14790 & 1.58516 & 0.14956 \\
\hline $\mathrm{H}$ & -0.66513 & 0.69760 & 0.11994 \\
\hline $\mathrm{C}$ & -0.97186 & 2.72369 & 0.27835 \\
\hline $\mathrm{C}$ & -2.21190 & 2.56767 & 0.90712 \\
\hline $\mathrm{C}$ & -0.62307 & 3.97088 & -0.25605 \\
\hline $\mathrm{C}$ & -3.08663 & 3.64945 & 1.00935 \\
\hline $\mathrm{H}$ & -2.48056 & 1.60301 & 1.31869 \\
\hline $\mathrm{C}$ & -1.50138 & 5.04599 & -0.13197 \\
\hline $\mathrm{H}$ & 0.32706 & 4.10124 & -0.75448 \\
\hline $\mathrm{C}$ & -2.73686 & 4.89932 & 0.50103 \\
\hline
\end{tabular}




\begin{tabular}{|c|c|c|c|}
\hline $\mathrm{H}$ & -3.40721 & 5.74456 & 0.60368 \\
\hline $\mathrm{C}$ & -1.15161 & 6.37342 & -0.75221 \\
\hline $\mathrm{C}$ & -4.44236 & 3.45316 & 1.62875 \\
\hline $\mathrm{C}$ & -1.71638 & 6.51056 & -1.97604 \\
\hline $\mathrm{C}$ & 0.17782 & 6.52921 & -0.91160 \\
\hline $\mathrm{C}$ & -1.59829 & 7.40687 & -0.00246 \\
\hline $\mathrm{C}$ & -5.37563 & 3.12958 & 0.69475 \\
\hline $\mathrm{C}$ & -4.44805 & 2.45689 & 2.54126 \\
\hline $\mathrm{C}$ & -4.88648 & 4.57068 & 2.24233 \\
\hline $\mathrm{H}$ & 3.60615 & 0.89429 & 0.41732 \\
\hline $\mathrm{C}$ & 3.67957 & -0.18100 & -1.42033 \\
\hline $\mathrm{C}$ & 5.09088 & -0.04244 & -1.66304 \\
\hline $\mathrm{C}$ & 2.87479 & -0.46004 & -2.50115 \\
\hline $\mathrm{C}$ & 5.55596 & -0.25593 & -3.00422 \\
\hline $\mathrm{C}$ & 6.03918 & 0.30011 & -0.66944 \\
\hline $\mathrm{C}$ & 3.44362 & -0.63950 & -3.78815 \\
\hline $\mathrm{H}$ & 1.80494 & -0.57666 & -2.38859 \\
\hline $\mathrm{C}$ & 6.94169 & -0.13989 & -3.27616 \\
\hline $\mathrm{C}$ & 7.38860 & 0.40997 & -0.96807 \\
\hline $\mathrm{H}$ & 5.74099 & 0.52386 & 0.34799 \\
\hline $\mathrm{H}$ & 2.79034 & -0.87144 & -4.62860 \\
\hline $\mathrm{C}$ & 7.84780 & 0.18015 & -2.28957 \\
\hline $\mathrm{H}$ & 7.26272 & -0.30779 & -4.29930 \\
\hline
\end{tabular}




$\begin{array}{lrrr}\mathrm{H} & 8.90085 & 0.26366 & -2.53221 \\ \mathrm{~N} & 4.72913 & -0.55725 & -4.04790 \\ \mathrm{O} & 8.20484 & 0.74425 & 0.07258 \\ \mathrm{C} & 9.58794 & 0.94407 & -0.17971 \\ \mathrm{H} & 9.75409 & 1.74532 & -0.91106 \\ \mathrm{H} & 10.02493 & 1.23515 & 0.77716 \\ \mathrm{H} & 10.07354 & 0.02470 & -0.53259 \\ \mathrm{H} & 1.97906 & -2.45065 & 0.23928 \\ \mathrm{O} & -2.81163 & 0.09574 & -1.09223 \\ \mathrm{C} & -3.65298 & -0.63341 & -0.58206 \\ \mathrm{C} & -3.40378 & -1.73326 & 0.36194 \\ \mathrm{C} & -4.60010 & -2.51327 & 0.88929 \\ \mathrm{H} & -5.32053 & -2.73114 & 0.09962 \\ \mathrm{C} & -2.26797 & -1.60311 & 1.25436 \\ \mathrm{H} & -2.51207 & -3.98969 & -0.53070 \\ \mathrm{O} & -1.25308 & -0.91601 & 1.02918 \\ \mathrm{O} & 0.96865 & -2.38265 & -0.99712 \\ \mathrm{~N} & -0.16403 & -2.95454 & -0.71422 \\ \mathrm{C} & -0.23019 & -3.77803 & 0.23300 \\ \mathrm{C} & -1.22272 & -2.64902 & -1.50850 \\ \mathrm{H} & -1.00113 & -1.92584 & -2.27592 \\ \mathrm{H} & -2.48804 & -3.17916 & -1.24908 \\ \mathrm{H} & -3.19727 & -2.25830\end{array}$




\begin{tabular}{|c|c|c|c|}
\hline $\mathrm{C}$ & -3.56428 & -2.33626 & -3.37425 \\
\hline $\mathrm{C}$ & -4.59280 & -4.14244 & -2.13218 \\
\hline $\mathrm{C}$ & -4.57063 & -2.42766 & -4.33141 \\
\hline $\mathrm{H}$ & -2.79031 & -1.58448 & -3.48624 \\
\hline $\mathrm{C}$ & -5.59818 & -4.23056 & -3.09354 \\
\hline $\mathrm{H}$ & -4.61128 & -4.80630 & -1.27226 \\
\hline $\mathrm{C}$ & -5.58978 & -3.37600 & -4.19725 \\
\hline $\mathrm{H}$ & -4.55918 & -1.75883 & -5.18779 \\
\hline $\mathrm{H}$ & -6.38699 & -4.96870 & -2.97908 \\
\hline $\mathrm{H}$ & -6.37136 & -3.44592 & -4.94886 \\
\hline $\mathrm{C}$ & -2.38687 & -2.35383 & 2.58309 \\
\hline $\mathrm{O}$ & -4.97975 & -0.49894 & -0.85001 \\
\hline $\mathrm{C}$ & -5.33294 & 0.58252 & -1.72350 \\
\hline $\mathrm{H}$ & -4.77612 & 0.52246 & -2.66129 \\
\hline $\mathrm{H}$ & -6.40273 & 0.46982 & -1.90565 \\
\hline $\mathrm{H}$ & -5.13044 & 1.54139 & -1.24038 \\
\hline $\mathrm{H}$ & -5.14882 & -1.92221 & 1.64516 \\
\hline $\mathrm{C}$ & -4.19263 & -3.82280 & 1.55223 \\
\hline $\mathrm{C}$ & -2.93905 & -3.77476 & 2.41945 \\
\hline $\mathrm{H}$ & -1.41092 & -2.35322 & 3.07444 \\
\hline $\mathrm{H}$ & -3.06317 & -1.76944 & 3.22628 \\
\hline $\mathrm{H}$ & -2.18746 & -4.41263 & 1.93719 \\
\hline $\mathrm{O}$ & -4.81278 & -4.85792 & 1.38584 \\
\hline
\end{tabular}


$\begin{array}{llll}\mathrm{H} & -3.17002 & -4.23993 & 3.38521\end{array}$

TSI-8 (S,S)

\begin{tabular}{|c|c|c|c|}
\hline $\mathrm{C}$ & 3.10447 & -1.30197 & 1.32181 \\
\hline $\mathrm{C}$ & 2.99543 & -1.04559 & 3.82519 \\
\hline $\mathrm{C}$ & 3.62875 & -0.49131 & 2.53083 \\
\hline $\mathrm{H}$ & 3.75933 & -2.16121 & 1.16016 \\
\hline $\mathrm{H}$ & 4.72034 & -0.57756 & 2.56443 \\
\hline $\mathrm{H}$ & 3.40057 & 0.57162 & 2.40583 \\
\hline $\mathrm{C}$ & 2.08948 & -3.07787 & 2.66804 \\
\hline $\mathrm{H}$ & 1.12822 & -3.42856 & 3.05032 \\
\hline $\mathrm{H}$ & 2.52027 & -3.87239 & 2.05448 \\
\hline $\mathrm{C}$ & 3.03952 & -2.58901 & 3.78759 \\
\hline $\mathrm{H}$ & 2.72479 & -3.00901 & 4.74860 \\
\hline $\mathrm{H}$ & 4.06322 & -2.93505 & 3.60477 \\
\hline $\mathrm{H}$ & 3.52776 & -0.66905 & 4.70359 \\
\hline $\mathrm{C}$ & 1.50175 & -0.62086 & 3.88690 \\
\hline $\mathrm{H}$ & 1.00941 & -1.23274 & 4.65399 \\
\hline $\mathrm{C}$ & 0.88725 & -0.94622 & 2.50070 \\
\hline $\mathrm{H}$ & -0.10222 & -1.40188 & 2.55620 \\
\hline $\mathrm{H}$ & 0.80299 & -0.05607 & 1.88297 \\
\hline $\mathrm{N}$ & 1.78264 & -1.91857 & 1.75539 \\
\hline $\mathrm{C}$ & 1.29097 & 0.82668 & 4.25079 \\
\hline
\end{tabular}




\begin{tabular}{|c|c|c|c|}
\hline $\mathrm{H}$ & 1.69171 & 1.56683 & 3.55708 \\
\hline $\mathrm{C}$ & 0.64886 & 1.23879 & 5.34450 \\
\hline $\mathrm{H}$ & 0.52438 & 2.29462 & 5.56753 \\
\hline $\mathrm{H}$ & 0.21857 & 0.53715 & 6.05668 \\
\hline $\mathrm{C}$ & 3.05241 & -0.51778 & -0.03716 \\
\hline $\mathrm{N}$ & 1.79251 & 0.17248 & -0.25311 \\
\hline $\mathrm{H}$ & 0.97996 & -0.38966 & -0.53565 \\
\hline $\mathrm{C}$ & 1.64644 & 1.52696 & -0.14696 \\
\hline $\mathrm{S}$ & 2.92274 & 2.58074 & 0.19650 \\
\hline $\mathrm{N}$ & 0.34240 & 1.93102 & -0.29882 \\
\hline $\mathrm{H}$ & -0.39460 & 1.22843 & -0.20537 \\
\hline $\mathrm{C}$ & -0.13727 & 3.26121 & -0.32210 \\
\hline $\mathrm{C}$ & -1.31625 & 3.54726 & 0.37227 \\
\hline $\mathrm{C}$ & 0.48346 & 4.27056 & -1.07304 \\
\hline $\mathrm{C}$ & -1.87278 & 4.82715 & 0.30964 \\
\hline $\mathrm{H}$ & -1.80156 & 2.76528 & 0.94431 \\
\hline $\mathrm{C}$ & -0.06889 & 5.54875 & -1.09976 \\
\hline $\mathrm{H}$ & 1.38996 & 4.05903 & -1.62248 \\
\hline $\mathrm{C}$ & -1.25175 & 5.84055 & -0.41389 \\
\hline $\mathrm{H}$ & -1.67373 & 6.83798 & -0.44185 \\
\hline $\mathrm{C}$ & 0.56711 & 6.62569 & -1.94008 \\
\hline $\mathrm{C}$ & -3.18838 & 5.06766 & 0.99720 \\
\hline $\mathrm{C}$ & -0.06589 & 6.76087 & -3.13029 \\
\hline
\end{tabular}




\begin{tabular}{|c|c|c|c|}
\hline $\mathrm{C}$ & 1.86238 & 6.36821 & -2.20747 \\
\hline $\mathrm{C}$ & 0.50477 & 7.83052 & -1.32973 \\
\hline $\mathrm{C}$ & -4.21552 & 4.52527 & 0.28925 \\
\hline $\mathrm{C}$ & -3.22869 & 4.50155 & 2.22259 \\
\hline $\mathrm{C}$ & -3.46177 & 6.37976 & 1.14288 \\
\hline $\mathrm{H}$ & 3.79112 & 0.28451 & 0.06275 \\
\hline $\mathrm{C}$ & 3.48179 & -1.39536 & -1.21367 \\
\hline $\mathrm{C}$ & 4.86682 & -1.76103 & -1.34492 \\
\hline $\mathrm{C}$ & 2.60525 & -1.86200 & -2.16693 \\
\hline $\mathrm{C}$ & 5.22407 & -2.61057 & -2.44553 \\
\hline $\mathrm{C}$ & 5.89122 & -1.33035 & -0.46754 \\
\hline $\mathrm{C}$ & 3.07473 & -2.69009 & -3.21869 \\
\hline $\mathrm{H}$ & 1.54818 & -1.62849 & -2.12622 \\
\hline $\mathrm{C}$ & 6.57829 & -2.99608 & -2.60253 \\
\hline $\mathrm{C}$ & 7.20796 & -1.72432 & -0.64920 \\
\hline $\mathrm{H}$ & 5.68910 & -0.65646 & 0.35693 \\
\hline $\mathrm{H}$ & 2.36520 & -3.05584 & -3.96012 \\
\hline $\mathrm{C}$ & 7.55637 & -2.57319 & -1.72999 \\
\hline $\mathrm{H}$ & 6.81715 & -3.63822 & -3.44439 \\
\hline $\mathrm{H}$ & 8.58344 & -2.88522 & -1.88030 \\
\hline $\mathrm{N}$ & 4.32510 & -3.06706 & -3.36592 \\
\hline $\mathrm{O}$ & 8.10497 & -1.24682 & 0.26138 \\
\hline $\mathrm{C}$ & 9.47896 & -1.56497 & 0.09907 \\
\hline
\end{tabular}




\begin{tabular}{|c|c|c|c|}
\hline $\mathrm{H}$ & 9.86943 & -1.19706 & -0.85850 \\
\hline $\mathrm{H}$ & 9.99877 & -1.06103 & 0.91589 \\
\hline $\mathrm{H}$ & 9.65676 & -2.64610 & 0.17120 \\
\hline $\mathrm{H}$ & 1.24411 & -2.32756 & 0.92281 \\
\hline $\mathrm{O}$ & -2.24343 & 0.51270 & 0.33101 \\
\hline $\mathrm{C}$ & -3.17716 & -0.11826 & -0.14651 \\
\hline $\mathrm{C}$ & -3.79780 & -1.35844 & 0.37637 \\
\hline $\mathrm{C}$ & -5.31924 & -1.39167 & 0.24340 \\
\hline $\mathrm{H}$ & -5.64274 & -1.31031 & -0.79575 \\
\hline $\mathrm{C}$ & -3.22253 & -1.92296 & 1.59848 \\
\hline $\mathrm{O}$ & -2.01040 & -1.95828 & 1.82619 \\
\hline $\mathrm{C}$ & -1.75133 & -3.32283 & -0.66330 \\
\hline $\mathrm{C}$ & -2.90976 & -2.72895 & -1.20226 \\
\hline $\mathrm{C}$ & -4.19663 & -2.54400 & 2.60131 \\
\hline $\mathrm{O}$ & -3.82428 & 0.30227 & -1.26954 \\
\hline $\mathrm{C}$ & -3.39934 & 1.55192 & -1.83108 \\
\hline $\mathrm{H}$ & -2.33370 & 1.52890 & -2.07278 \\
\hline $\mathrm{H}$ & -3.98879 & 1.67534 & -2.74067 \\
\hline $\mathrm{H}$ & -3.59978 & 2.37510 & -1.14081 \\
\hline $\mathrm{H}$ & -5.75400 & -0.50824 & 0.74495 \\
\hline $\mathrm{C}$ & -6.04085 & -2.59154 & 0.85479 \\
\hline $\mathrm{C}$ & -5.30242 & -3.37078 & 1.93388 \\
\hline $\mathrm{H}$ & -3.61447 & -3.14410 & 3.30664 \\
\hline
\end{tabular}




\begin{tabular}{|c|c|c|c|}
\hline $\mathrm{H}$ & -4.65083 & -1.72264 & 3.17705 \\
\hline $\mathrm{H}$ & -4.86819 & -4.25465 & 1.44729 \\
\hline $\mathrm{O}$ & -7.17126 & -2.88012 & 0.51409 \\
\hline $\mathrm{H}$ & -6.03656 & -3.74389 & 2.65554 \\
\hline $\mathrm{C}$ & -4.04377 & -3.55361 & -1.65792 \\
\hline $\mathrm{C}$ & -4.91350 & -3.02673 & -2.63172 \\
\hline $\mathrm{C}$ & -4.28015 & -4.86372 & -1.19815 \\
\hline $\mathrm{C}$ & -5.98640 & -3.77178 & -3.11700 \\
\hline $\mathrm{H}$ & -4.73679 & -2.02173 & -3.00552 \\
\hline $\mathrm{C}$ & -5.35196 & -5.60797 & -1.68380 \\
\hline $\mathrm{H}$ & -3.62013 & -5.30917 & -0.45953 \\
\hline $\mathrm{C}$ & -6.21233 & -5.06431 & -2.64180 \\
\hline $\mathrm{H}$ & -6.64600 & -3.34234 & -3.86574 \\
\hline $\mathrm{H}$ & -5.51734 & -6.61631 & -1.31441 \\
\hline $\mathrm{H}$ & -7.05072 & -5.64564 & -3.01461 \\
\hline $\mathrm{H}$ & -2.72549 & -1.85234 & -1.81004 \\
\hline $\mathrm{H}$ & -1.70501 & -4.30277 & -0.21501 \\
\hline $\mathrm{N}$ & -0.58444 & -2.64721 & -0.59309 \\
\hline $\mathrm{O}$ & -0.47151 & -1.46484 & -1.04537 \\
\hline $\mathrm{O}$ & 0.43386 & -3.23814 & -0.07351 \\
\hline
\end{tabular}

TSII-1 (S,S)
C
2.46414
1.25087
0.28308 


\begin{tabular}{|c|c|c|c|}
\hline $\mathrm{C}$ & 2.90235 & 3.43116 & 1.46632 \\
\hline $\mathrm{C}$ & 3.51274 & 2.35182 & 0.54849 \\
\hline $\mathrm{H}$ & 2.55613 & 0.45973 & 1.03113 \\
\hline $\mathrm{H}$ & 4.39476 & 1.90403 & 1.01642 \\
\hline $\mathrm{H}$ & 3.83041 & 2.78634 & -0.40363 \\
\hline $\mathrm{C}$ & 0.95744 & 1.96564 & 2.08324 \\
\hline $\mathrm{H}$ & 0.00623 & 2.46304 & 2.26926 \\
\hline $\mathrm{H}$ & 0.87372 & 0.94646 & 2.46061 \\
\hline $\mathrm{C}$ & 2.18053 & 2.73418 & 2.63809 \\
\hline $\mathrm{H}$ & 1.84937 & 3.46971 & 3.37938 \\
\hline $\mathrm{H}$ & 2.86992 & 2.05179 & 3.14861 \\
\hline $\mathrm{H}$ & 3.68297 & 4.10061 & 1.84076 \\
\hline $\mathrm{C}$ & 1.83970 & 4.25144 & 0.68246 \\
\hline $\mathrm{H}$ & 1.25724 & 4.82519 & 1.41535 \\
\hline $\mathrm{C}$ & 0.91670 & 3.22930 & -0.02934 \\
\hline $\mathrm{H}$ & -0.14367 & 3.46796 & 0.06258 \\
\hline $\mathrm{H}$ & 1.16332 & 3.13343 & -1.08339 \\
\hline $\mathrm{N}$ & 1.09651 & 1.86177 & 0.58540 \\
\hline $\mathrm{C}$ & 2.42724 & 5.22575 & -0.30580 \\
\hline $\mathrm{H}$ & 2.96318 & 4.78955 & -1.14929 \\
\hline $\mathrm{C}$ & 2.31944 & 6.55181 & -0.21263 \\
\hline $\mathrm{H}$ & 2.76602 & 7.21395 & -0.94924 \\
\hline $\mathrm{H}$ & 1.77800 & 7.02801 & 0.60284 \\
\hline
\end{tabular}




\begin{tabular}{|c|c|c|c|}
\hline $\mathrm{C}$ & 2.55663 & 0.54858 & -1.10288 \\
\hline $\mathrm{C}$ & 3.87209 & -0.21967 & -1.13115 \\
\hline $\mathrm{C}$ & 3.99741 & -1.50480 & -0.50044 \\
\hline $\mathrm{C}$ & 4.98967 & 0.29829 & -1.75196 \\
\hline $\mathrm{C}$ & 5.27972 & -2.14799 & -0.56608 \\
\hline $\mathrm{C}$ & 2.93573 & -2.16704 & 0.16359 \\
\hline $\mathrm{C}$ & 6.20793 & -0.42582 & -1.75294 \\
\hline $\mathrm{H}$ & 4.94551 & 1.26507 & -2.24276 \\
\hline $\mathrm{C}$ & 5.43759 & -3.42101 & 0.03506 \\
\hline $\mathrm{C}$ & 3.12600 & -3.41405 & 0.73669 \\
\hline $\mathrm{H}$ & 1.94687 & -1.72646 & 0.23690 \\
\hline $\mathrm{H}$ & 7.08171 & -0.00072 & -2.24664 \\
\hline $\mathrm{C}$ & 4.39218 & -4.04948 & 0.67444 \\
\hline $\mathrm{H}$ & 6.41622 & -3.88584 & -0.03087 \\
\hline $\mathrm{H}$ & 4.54473 & -5.02566 & 1.12014 \\
\hline $\mathrm{N}$ & 6.36751 & -1.60122 & -1.18555 \\
\hline $\mathrm{O}$ & 2.03336 & -3.96838 & 1.34530 \\
\hline $\mathrm{C}$ & 2.15269 & -5.26050 & 1.91977 \\
\hline $\mathrm{H}$ & 2.89245 & -5.27776 & 2.73091 \\
\hline $\mathrm{H}$ & 1.16847 & -5.49643 & 2.32862 \\
\hline $\mathrm{H}$ & 2.42128 & -6.01512 & 1.16911 \\
\hline $\mathrm{N}$ & 2.44113 & 1.50203 & -2.21394 \\
\hline $\mathrm{H}$ & 2.86984 & 1.08144 & -3.03553 \\
\hline
\end{tabular}




$\begin{array}{llll}\mathrm{H} & 1.44098 & 1.52355 & -2.43431 \\ \mathrm{H} & 1.74009 & -0.18073 & -1.15226 \\ \mathrm{O} & 0.01073 & -0.48641 & -2.93875 \\ \mathrm{~N} & -0.78751 & 0.08361 & -2.14880 \\ \mathrm{O} & -0.45262 & 1.20203 & -1.60020 \\ \mathrm{C} & -2.00860 & -0.46038 & -1.90640 \\ \mathrm{H} & -2.20068 & -1.36119 & -2.46992 \\ \mathrm{C} & -2.91766 & 0.16860 & -1.00183 \\ \mathrm{H} & -2.55442 & 1.15855 & -0.74352 \\ \mathrm{C} & -4.37799 & 0.14197 & -1.30712 \\ \mathrm{C} & -5.00170 & -0.91658 & -1.98833 \\ \mathrm{C} & -5.16792 & 1.24179 & -0.92956 \\ \mathrm{C} & -6.36519 & -0.87874 & -2.27485 \\ \mathrm{H} & -4.42267 & -1.78070 & -2.29789 \\ \mathrm{H} & -0.38876 & -0.53243 & 0.88779 \\ \mathrm{H} & -6.53050 & 1.28573 & -1.22215 \\ \mathrm{H} & -4.69938 & 2.07797 & -0.41665 \\ \mathrm{H} & -7.13576 & 0.22230 & -1.89450 \\ \mathrm{H} & -6.82602 & -1.71291 & -2.79644 \\ \mathrm{H} & -7.11782 & 2.15214 & -0.92918 \\ \mathrm{H} & -8.19785 & 0.25145 & -2.12225 \\ \mathrm{H} & 0.34345 & 1.24834 & 0.20870 \\ \mathrm{H} & -1.14345 & 0.96470\end{array}$




\begin{tabular}{|c|c|c|c|}
\hline $\mathrm{C}$ & -1.42366 & -2.66196 & 1.11208 \\
\hline 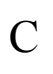 & -2.80048 & -0.50723 & 0.90217 \\
\hline $\mathrm{H}$ & -1.61708 & -2.89227 & 2.17182 \\
\hline 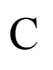 & -2.47857 & -3.35743 & 0.24371 \\
\hline $\mathrm{C}$ & -3.95594 & -1.46925 & 1.21209 \\
\hline $\mathrm{H}$ & -2.47150 & -4.44453 & 0.37307 \\
\hline $\mathrm{H}$ & -4.92275 & -1.02781 & 0.96853 \\
\hline 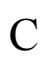 & -3.87691 & -2.83715 & 0.54096 \\
\hline $\mathrm{O}$ & -4.87353 & -3.48970 & 0.29771 \\
\hline I & -2.26652 & -3.16631 & -0.81706 \\
\hline $\mathrm{H}$ & -0.41154 & -3.00930 & 0.89089 \\
\hline $\mathrm{H}$ & -3.99249 & -1.67083 & 2.29575 \\
\hline $\mathrm{C}$ & -2.88205 & 0.79745 & 1.61526 \\
\hline $\mathrm{O}$ & -2.07189 & 1.71233 & 1.56218 \\
\hline $\mathrm{O}$ & -4.02988 & 0.91326 & 2.33704 \\
\hline $\mathrm{C}$ & -4.21879 & 2.16660 & 3.00359 \\
\hline $\mathrm{H}$ & -4.20128 & 2.99426 & 2.28886 \\
\hline $\mathrm{H}$ & -5.19518 & 2.09729 & 3.48476 \\
\hline $\mathrm{H}$ & -3.43655 & 2.33097 & 3.75053 \\
\hline
\end{tabular}

TSII-2 (R,S)
C
2.31198
1.45695
0.48257
$\begin{array}{llll}\text { C } & 2.22254 & 3.76677 & 1.49531\end{array}$ 


\begin{tabular}{|c|c|c|c|}
\hline $\mathrm{C}$ & 3.08540 & 2.77813 & 0.69114 \\
\hline $\mathrm{H}$ & 2.63618 & 0.70654 & 1.20810 \\
\hline $\mathrm{H}$ & 4.02174 & 2.57416 & 1.22033 \\
\hline $\mathrm{H}$ & 3.33749 & 3.19951 & -0.28505 \\
\hline $\mathrm{C}$ & 0.80854 & 1.88729 & 2.38242 \\
\hline $\mathrm{H}$ & -0.24026 & 2.04588 & 2.63291 \\
\hline $\mathrm{H}$ & 1.11633 & 0.93822 & 2.82756 \\
\hline $\mathrm{C}$ & 1.72847 & 3.06718 & 2.77761 \\
\hline $\mathrm{H}$ & 1.18014 & 3.77084 & 3.41358 \\
\hline $\mathrm{H}$ & 2.58541 & 2.70839 & 3.35868 \\
\hline $\mathrm{H}$ & 2.80866 & 4.65473 & 1.75227 \\
\hline $\mathrm{C}$ & 0.98266 & 4.19946 & 0.66193 \\
\hline $\mathrm{H}$ & 0.33402 & 4.78375 & 1.32779 \\
\hline $\mathrm{C}$ & 0.23302 & 2.90896 & 0.22726 \\
\hline $\mathrm{H}$ & -0.81084 & 2.90300 & 0.54435 \\
\hline $\mathrm{H}$ & 0.24894 & 2.74443 & -0.84964 \\
\hline $\mathrm{N}$ & 0.85711 & 1.70486 & 0.88644 \\
\hline $\mathrm{C}$ & 1.32553 & 5.06138 & -0.52779 \\
\hline $\mathrm{H}$ & 1.81963 & 4.55417 & -1.35603 \\
\hline $\mathrm{C}$ & 1.04799 & 6.36309 & -0.61312 \\
\hline $\mathrm{H}$ & 1.31787 & 6.94713 & -1.48867 \\
\hline $\mathrm{H}$ & 0.53518 & 6.89614 & 0.18596 \\
\hline $\mathrm{C}$ & 2.49927 & 0.80521 & -0.92779 \\
\hline
\end{tabular}




$\begin{array}{llll}\mathrm{C} & 3.90503 & 0.20636 & -0.93472 \\ \mathrm{C} & 4.17721 & -1.07418 & -0.34131 \\ \mathrm{C} & 4.96804 & 0.89119 & -1.48646 \\ \mathrm{C} & 5.53503 & -1.54151 & -0.37630 \\ \mathrm{C} & 3.19089 & -1.89078 & 0.26399 \\ \mathrm{C} & 6.27039 & 0.33223 & -1.46362 \\ \mathrm{H} & 4.81969 & 1.86566 & -1.93993 \\ \mathrm{C} & 5.83838 & -2.80315 & 0.19236 \\ \mathrm{C} & 3.52464 & -3.11999 & 0.81109 \\ \mathrm{H} & 2.15015 & -1.58743 & 0.30769 \\ \mathrm{H} & 7.09749 & 0.88800 & -1.90502 \\ \mathrm{C} & 4.86472 & -3.58301 & 0.77610 \\ \mathrm{H} & 6.87137 & -3.13366 & 0.14931 \\ \mathrm{H} & 5.13076 & -4.54462 & 1.19998 \\ \mathrm{H} & 2.87510 & 1.47245 & -2.81358 \\ \mathrm{~N} & 6.56263 & -0.83490 & -0.93355 \\ \mathrm{H} & 2.49505 & -3.82769 & 1.36641 \\ \mathrm{H} & 2.76926 & -5.09983 & 1.93202 \\ \mathrm{H} & 3.47843 & -5.02912 & 2.76737 \\ \mathrm{H} & 1.81341 & -5.47218 & 2.30535 \\ \mathrm{H} & 3.16143 & -5.80160 & 1.18424 \\ \mathrm{H} & 2.31093 & 1.76582 & -2.02057 \\ \mathrm{H} & & 1.70042 & -2.34916\end{array}$




\begin{tabular}{|c|c|c|c|}
\hline $\mathrm{H}$ & 1.77617 & -0.01549 & -0.99635 \\
\hline $\mathrm{C}$ & -2.35192 & 0.89614 & -1.56250 \\
\hline $\mathrm{C}$ & -3.18793 & -0.18328 & -1.17626 \\
\hline $\mathrm{H}$ & 0.28569 & 0.84989 & 0.66211 \\
\hline $\mathrm{O}$ & -0.06449 & -0.74217 & 0.32407 \\
\hline $\mathrm{C}$ & -1.00352 & -1.50464 & 0.03812 \\
\hline $\mathrm{C}$ & -0.66742 & -2.86221 & -0.56509 \\
\hline $\mathrm{C}$ & -2.41969 & -1.23502 & 0.36502 \\
\hline $\mathrm{H}$ & -0.45528 & -3.53707 & 0.27930 \\
\hline $\mathrm{C}$ & -1.76885 & -3.44392 & -1.45463 \\
\hline $\mathrm{C}$ & -3.27567 & -2.50540 & 0.42548 \\
\hline $\mathrm{H}$ & -1.55523 & -4.47644 & -1.75174 \\
\hline $\mathrm{H}$ & -4.33097 & -2.27273 & 0.57100 \\
\hline $\mathrm{C}$ & -3.14193 & -3.40013 & -0.80318 \\
\hline $\mathrm{O}$ & -4.08381 & -4.04246 & -1.23099 \\
\hline $\mathrm{H}$ & -1.82144 & -2.85500 & -2.37933 \\
\hline $\mathrm{H}$ & 0.26724 & -2.75477 & -1.11879 \\
\hline $\mathrm{H}$ & -2.97979 & -3.11961 & 1.29249 \\
\hline $\mathrm{C}$ & -2.60965 & -0.27368 & 1.47834 \\
\hline $\mathrm{O}$ & -2.01462 & 0.78763 & 1.62314 \\
\hline $\mathrm{O}$ & -3.57318 & -0.67476 & 2.34455 \\
\hline $\mathrm{C}$ & -3.88336 & 0.23707 & 3.40418 \\
\hline $\mathrm{H}$ & -4.27780 & 1.17362 & 3.00094 \\
\hline
\end{tabular}




\begin{tabular}{|c|c|c|c|}
\hline $\mathrm{H}$ & -4.64224 & -0.26380 & 4.00651 \\
\hline $\mathrm{H}$ & -2.99580 & 0.45010 & 4.00671 \\
\hline $\mathrm{C}$ & -4.58819 & 0.15300 & -0.77490 \\
\hline $\mathrm{C}$ & -4.90343 & 1.32210 & -0.05874 \\
\hline $\mathrm{C}$ & -5.64048 & -0.69672 & -1.15507 \\
\hline $\mathrm{C}$ & -6.22524 & 1.63171 & 0.25662 \\
\hline $\mathrm{H}$ & -4.10801 & 1.98879 & 0.25871 \\
\hline $\mathrm{C}$ & -6.96408 & -0.38627 & -0.83844 \\
\hline $\mathrm{H}$ & -5.42136 & -1.60977 & -1.70185 \\
\hline $\mathrm{C}$ & -7.26239 & 0.77963 & -0.13288 \\
\hline $\mathrm{H}$ & -6.44745 & 2.54469 & 0.80345 \\
\hline $\mathrm{H}$ & -7.76043 & -1.05780 & -1.14762 \\
\hline $\mathrm{H}$ & -8.29268 & 1.02450 & 0.11105 \\
\hline $\mathrm{H}$ & -3.13459 & -1.02742 & -1.85772 \\
\hline $\mathrm{H}$ & -2.53806 & 1.93224 & -1.33213 \\
\hline $\mathrm{N}$ & -1.22105 & 0.66761 & -2.28456 \\
\hline $\mathrm{O}$ & -0.87920 & -0.51305 & -2.58356 \\
\hline $\mathrm{O}$ & -0.52165 & 1.67209 & -2.65013 \\
\hline
\end{tabular}

TSII-3 (S,R)
$\begin{array}{llll}\mathrm{C} & 2.40552 & 1.27478 & 0.32592\end{array}$
$\begin{array}{llll}\text { C } & 2.66208 & 3.41274 & 1.62817\end{array}$
$\begin{array}{llll}\text { C } & 3.34581 & 2.46166 & 0.62418\end{array}$ 


\begin{tabular}{|c|c|c|c|}
\hline $\mathrm{H}$ & 2.59509 & 0.46725 & 1.03662 \\
\hline $\mathrm{H}$ & 4.28538 & 2.08011 & 1.03533 \\
\hline $\mathrm{H}$ & 3.58311 & 2.98113 & -0.30893 \\
\hline $\mathrm{C}$ & 0.87999 & 1.73649 & 2.18979 \\
\hline $\mathrm{H}$ & -0.10247 & 2.14625 & 2.41991 \\
\hline $\mathrm{H}$ & 0.88707 & 0.69312 & 2.50716 \\
\hline $\mathrm{C}$ & 2.04762 & 2.57134 & 2.76623 \\
\hline $\mathrm{H}$ & 1.67547 & 3.21849 & 3.56793 \\
\hline $\mathrm{H}$ & 2.81270 & 1.92182 & 3.20742 \\
\hline $\mathrm{H}$ & 3.38429 & 4.13082 & 2.02877 \\
\hline $\mathrm{C}$ & 1.50058 & 4.17174 & 0.92644 \\
\hline $\mathrm{H}$ & 0.87913 & 4.62627 & 1.70905 \\
\hline $\mathrm{C}$ & 0.67077 & 3.11172 & 0.15713 \\
\hline $\mathrm{H}$ & -0.40586 & 3.23658 & 0.27316 \\
\hline $\mathrm{H}$ & 0.91225 & 3.10571 & -0.90264 \\
\hline $\mathrm{N}$ & 0.99233 & 1.73192 & 0.68436 \\
\hline $\mathrm{C}$ & 1.95822 & 5.27107 & 0.00328 \\
\hline $\mathrm{H}$ & 2.52459 & 4.95712 & -0.87380 \\
\hline $\mathrm{C}$ & 1.70474 & 6.56703 & 0.19038 \\
\hline $\mathrm{H}$ & 2.06117 & 7.32438 & -0.50233 \\
\hline $\mathrm{H}$ & 1.12795 & 6.92152 & 1.04272 \\
\hline $\mathrm{C}$ & 2.54104 & 0.64577 & -1.09070 \\
\hline $\mathrm{C}$ & 3.93830 & 0.04197 & -1.17824 \\
\hline
\end{tabular}




\begin{tabular}{|c|c|c|c|}
\hline $\mathrm{C}$ & 4.25222 & -1.21056 & -0.54745 \\
\hline $\mathrm{C}$ & 4.95508 & 0.68894 & -1.84908 \\
\hline $\mathrm{C}$ & 5.60259 & -1.68572 & -0.65975 \\
\hline $\mathrm{C}$ & 3.31336 & -1.99294 & 0.16858 \\
\hline $\mathrm{C}$ & 6.25295 & 0.12208 & -1.90177 \\
\hline $\mathrm{H}$ & 4.77044 & 1.64045 & -2.33642 \\
\hline $\mathrm{C}$ & 5.94858 & -2.91462 & -0.04549 \\
\hline $\mathrm{C}$ & 3.68815 & -3.18881 & 0.75994 \\
\hline $\mathrm{H}$ & 2.27809 & -1.68514 & 0.26682 \\
\hline $\mathrm{H}$ & 7.04345 & 0.64690 & -2.43799 \\
\hline $\mathrm{C}$ & 5.02248 & -3.65658 & 0.65344 \\
\hline $\mathrm{H}$ & 6.97554 & -3.25084 & -0.14752 \\
\hline $\mathrm{H}$ & 5.31981 & -4.59303 & 1.11134 \\
\hline $\mathrm{N}$ & 6.58459 & -1.01602 & -1.33281 \\
\hline $\mathrm{O}$ & 2.70365 & -3.86219 & 1.42972 \\
\hline $\mathrm{C}$ & 3.01640 & -5.10651 & 2.03708 \\
\hline $\mathrm{H}$ & 3.78413 & -4.99653 & 2.81405 \\
\hline $\mathrm{H}$ & 2.08948 & -5.45435 & 2.49657 \\
\hline $\mathrm{H}$ & 3.35256 & -5.84553 & 1.29820 \\
\hline $\mathrm{N}$ & 2.29210 & 1.62531 & -2.15442 \\
\hline $\mathrm{H}$ & 2.70500 & 1.26709 & -3.01309 \\
\hline $\mathrm{H}$ & 1.28151 & 1.57884 & -2.32065 \\
\hline $\mathrm{H}$ & 1.81080 & -0.17160 & -1.15367 \\
\hline
\end{tabular}




\begin{tabular}{|c|c|c|c|}
\hline $\mathrm{O}$ & -0.00615 & -0.61998 & -2.85141 \\
\hline $\mathrm{N}$ & -0.85341 & -0.00882 & -2.14099 \\
\hline $\mathrm{O}$ & -0.53866 & 1.10777 & -1.58325 \\
\hline $\mathrm{C}$ & -2.10441 & -0.51534 & -1.98981 \\
\hline $\mathrm{H}$ & -2.27764 & -1.42555 & -2.54354 \\
\hline $\mathrm{C}$ & -3.02811 & 0.07118 & -1.07419 \\
\hline $\mathrm{H}$ & -2.71987 & 1.06841 & -0.77522 \\
\hline $\mathrm{C}$ & -4.47888 & -0.04534 & -1.38992 \\
\hline $\mathrm{C}$ & -5.04852 & -1.20405 & -1.94555 \\
\hline $\mathrm{C}$ & -5.31268 & 1.06613 & -1.17750 \\
\hline $\mathrm{C}$ & -6.40552 & -1.25386 & -2.25868 \\
\hline $\mathrm{H}$ & -4.42712 & -2.07506 & -2.13192 \\
\hline $\mathrm{C}$ & -6.66890 & 1.02171 & -1.50110 \\
\hline $\mathrm{H}$ & -4.88288 & 1.97980 & -0.77271 \\
\hline $\mathrm{C}$ & -7.22206 & -0.14271 & -2.03671 \\
\hline $\mathrm{H}$ & -6.82709 & -2.16364 & -2.67707 \\
\hline $\mathrm{H}$ & -7.29183 & 1.89735 & -1.33800 \\
\hline $\mathrm{H}$ & -8.27955 & -0.18372 & -2.28236 \\
\hline $\mathrm{H}$ & 0.28717 & 1.07614 & 0.29255 \\
\hline $\mathrm{O}$ & -0.37723 & -0.80333 & 0.76531 \\
\hline $\mathrm{C}$ & -1.47598 & -1.32728 & 0.61527 \\
\hline $\mathrm{C}$ & -2.81332 & -0.70234 & 0.81173 \\
\hline $\mathrm{C}$ & -3.91121 & -1.72887 & 1.12625 \\
\hline
\end{tabular}




$\begin{array}{llll}\mathrm{H} & -3.57708 & -2.38585 & 1.94772 \\ \mathrm{C} & -2.81541 & 0.51203 & 1.66655 \\ \mathrm{O} & -2.03490 & 1.45296 & 1.51215 \\ \mathrm{O} & -1.59776 & -2.59748 & 0.18012 \\ \mathrm{C} & -0.38886 & -3.24575 & -0.25791 \\ \mathrm{H} & 0.05018 & -2.68864 & -1.09044 \\ \mathrm{H} & 0.33137 & -3.32921 & 0.55850 \\ \mathrm{H} & -0.70380 & -4.23657 & -0.58887 \\ \mathrm{H} & -4.08985 & -2.39947 & 0.28474 \\ \mathrm{C} & -3.87249 & 0.58254 & 2.76716 \\ \mathrm{H} & -3.55489 & -0.09311 & 3.57684 \\ \mathrm{H} & -3.87030 & 1.59807 & 3.17314 \\ \mathrm{C} & -5.26253 & -1.18769 & 1.58526 \\ \mathrm{C} & -5.26704 & 0.16826 & 2.27968 \\ \mathrm{H} & -5.99492 & 0.13659 & 3.09735 \\ \mathrm{H} & -5.64659 & 0.89753 & 1.55283 \\ \mathrm{O} & -6.28000 & -1.83875 & 1.45155\end{array}$

TSII-4 (R,R)

$\begin{array}{llll}\mathrm{C} & 2.33803 & 1.32085 & 0.61509 \\ \mathrm{C} & 2.22210 & 3.47289 & 1.92689 \\ \mathrm{C} & 3.09579 & 2.61060 & 0.99940 \\ \mathrm{H} & 2.66481 & 0.48912 & 1.24412\end{array}$




\begin{tabular}{|c|c|c|c|}
\hline $\mathrm{H}$ & 4.03372 & 2.34657 & 1.49829 \\
\hline $\mathrm{H}$ & 3.34595 & 3.16010 & 0.08880 \\
\hline $\mathrm{C}$ & 0.81789 & 1.47805 & 2.54078 \\
\hline $\mathrm{H}$ & -0.23366 & 1.59509 & 2.80052 \\
\hline $\mathrm{H}$ & 1.13198 & 0.47958 & 2.85455 \\
\hline $\mathrm{C}$ & 1.72645 & 2.60006 & 3.09776 \\
\hline $\mathrm{H}$ & 1.16814 & 3.20584 & 3.81982 \\
\hline $\mathrm{H}$ & 2.58320 & 2.17273 & 3.63114 \\
\hline $\mathrm{H}$ & 2.80026 & 4.32107 & 2.30661 \\
\hline $\mathrm{C}$ & 0.98411 & 4.00523 & 1.15156 \\
\hline $\mathrm{H}$ & 0.32630 & 4.48991 & 1.88492 \\
\hline $\mathrm{C}$ & 0.24703 & 2.78298 & 0.53895 \\
\hline $\mathrm{H}$ & -0.79740 & 2.72316 & 0.84395 \\
\hline $\mathrm{H}$ & 0.27086 & 2.76873 & -0.55001 \\
\hline $\mathrm{N}$ & 0.87695 & 1.50022 & 1.03161 \\
\hline $\mathrm{C}$ & 1.33005 & 5.02354 & 0.09312 \\
\hline $\mathrm{H}$ & 1.83242 & 4.63745 & -0.79350 \\
\hline $\mathrm{C}$ & 1.04605 & 6.32339 & 0.18481 \\
\hline $\mathrm{H}$ & 1.31858 & 7.02323 & -0.60034 \\
\hline $\mathrm{H}$ & 0.52456 & 6.73926 & 1.04547 \\
\hline $\mathrm{C}$ & 2.55151 & 0.85408 & -0.86185 \\
\hline $\mathrm{C}$ & 3.98259 & 0.32041 & -0.93027 \\
\hline $\mathrm{C}$ & 4.31922 & -0.99244 & -0.45355 \\
\hline
\end{tabular}




$\begin{array}{llll}\mathrm{C} & 5.00869 & 1.09840 & -1.42542 \\ \mathrm{C} & 5.69597 & -1.39291 & -0.53593 \\ \mathrm{C} & 3.37697 & -1.90356 & 0.08177 \\ \mathrm{C} & 6.33529 & 0.60005 & -1.45793 \\ \mathrm{H} & 4.81151 & 2.10117 & -1.78891 \\ \mathrm{C} & 6.06112 & -2.68428 & -0.08169 \\ \mathrm{C} & 3.76971 & -3.16049 & 0.51391 \\ \mathrm{H} & 2.32494 & -1.65128 & 0.15650 \\ \mathrm{H} & 7.13208 & 1.23000 & -1.85282 \\ \mathrm{C} & 5.12861 & -3.55715 & 0.43349 \\ \mathrm{H} & 7.10775 & -2.96152 & -0.15776 \\ \mathrm{H} & 5.44013 & -4.53961 & 0.76894 \\ \mathrm{~N} & 6.68548 & -0.59408 & -1.03378 \\ \mathrm{O} & 2.77471 & -3.96314 & 0.99977 \\ \mathrm{C} & 3.10293 & -5.27430 & 1.43500 \\ \mathrm{H} & 1.86149 & 0.01775 & -1.03259 \\ \mathrm{H} & 3.80851 & -5.25710 & 2.27582 \\ \mathrm{H} & 2.16344 & -5.72145 & 1.76457 \\ \mathrm{H} & 3.52433 & -5.87847 & 0.62114 \\ \mathrm{H} & 2.33495 & 1.93162 & -1.83005 \\ \mathrm{H} & 2.87416 & 1.72781 & -2.66756 \\ \mathrm{H} & 1.35535 & 1.91544 & -2.14173 \\ \mathrm{H} & & 1.01073 & -1.54390\end{array}$




$\begin{array}{lrrr}\mathrm{C} & -3.12198 & -0.11832 & -1.30025 \\ \mathrm{H} & 0.30633 & 0.69068 & 0.69388 \\ \mathrm{O} & -0.12247 & -0.94116 & 0.17162 \\ \mathrm{C} & -1.15152 & -1.54880 & -0.13390 \\ \mathrm{C} & -2.54399 & -1.22251 & 0.27919 \\ \mathrm{C} & -3.45117 & -2.46181 & 0.30526 \\ \mathrm{H} & -2.92010 & -3.30132 & 0.78122 \\ \mathrm{C} & -2.64249 & -0.30924 & 1.43323 \\ \mathrm{O} & -1.94090 & 0.70179 & 1.54635 \\ \mathrm{C} & -4.56921 & 0.18625 & -1.07831 \\ \mathrm{C} & -4.99018 & 1.29873 & -0.32621 \\ \mathrm{C} & -5.55134 & -0.62189 & -1.67193 \\ \mathrm{C} & -6.34532 & 1.58815 & -0.17650 \\ \mathrm{H} & -4.25111 & 1.93227 & 0.15435 \\ \mathrm{H} & -2.54721 & 2.01777 & -1.25434 \\ \mathrm{H} & -6.90919 & -0.33606 & -1.51836 \\ \mathrm{H} & -5.24802 & -1.47892 & -2.26711 \\ \mathrm{H} & -7.31208 & 0.77026 & -0.77041 \\ \mathrm{H} & -6.64871 & 2.45441 & 0.40590 \\ \mathrm{H} & -7.64954 & -0.98017 & -1.98438 \\ \mathrm{H} & -8.36820 & 0.99613 & -0.65140 \\ \mathrm{H} & -2.95009 & -0.94167 & -1.99146 \\ \mathrm{H} & -2.24907 \\ \mathrm{H} & & & 72\end{array}$




$\begin{array}{llll}\mathrm{O} & -0.71625 & -0.19199 & -2.68441 \\ \mathrm{O} & -0.48328 & 1.99593 & -2.47081 \\ \mathrm{H} & -3.66463 & -2.81841 & -0.70659 \\ \mathrm{O} & -1.12126 & -2.65846 & -0.88817 \\ \mathrm{C} & 0.13779 & -3.01954 & -1.48047 \\ \mathrm{H} & -0.09274 & -3.85190 & -2.14703 \\ \mathrm{H} & 0.53010 & -2.17317 & -2.04677 \\ \mathrm{H} & 0.84543 & -3.33696 & -0.71091 \\ \mathrm{C} & -4.78271 & -2.37360 & 1.04824 \\ \mathrm{C} & -3.63838 & -0.65109 & 2.54015 \\ \mathrm{H} & -3.12540 & -1.37073 & 3.19876 \\ \mathrm{H} & -3.80026 & 0.25327 & 3.13442 \\ \mathrm{C} & -4.96390 & -1.25950 & 2.06577 \\ \mathrm{H} & -5.55126 & -1.65307 & 2.90231 \\ \mathrm{H} & -5.58186 & -0.49042 & 1.58691 \\ \mathrm{O} & -5.64890 & -3.20512 & 0.85391\end{array}$

TSII-5 (R,R)
$\begin{array}{llll}\text { C } & -2.30355 & 1.10727 & -0.42401\end{array}$
$\begin{array}{llll}\text { C } & -2.57246 & 3.23972 & -1.73164\end{array}$
$\begin{array}{llll}\text { C } & -3.25429 & 2.28386 & -0.72768\end{array}$
$\mathrm{H} \quad-2.46852 \quad 0.30130 \quad-1.14113$
$\begin{array}{llll}\mathrm{H} & -4.18813 & 1.89176 & -1.14141\end{array}$ 


\begin{tabular}{|c|c|c|c|}
\hline $\mathrm{H}$ & -3.50994 & 2.81057 & 0.19699 \\
\hline $\mathrm{C}$ & -0.74018 & 1.60467 & -2.25020 \\
\hline $\mathrm{H}$ & 0.23605 & 2.04965 & -2.45053 \\
\hline $\mathrm{H}$ & -0.71250 & 0.56133 & -2.56616 \\
\hline $\mathrm{C}$ & -1.91677 & 2.40757 & -2.85493 \\
\hline $\mathrm{H}$ & -1.54774 & 3.06117 & -3.65283 \\
\hline $\mathrm{H}$ & -2.65491 & 1.73491 & -3.30649 \\
\hline $\mathrm{H}$ & -3.30215 & 3.93967 & -2.15045 \\
\hline $\mathrm{C}$ & -1.43684 & 4.02266 & -1.01544 \\
\hline $\mathrm{H}$ & -0.80105 & 4.47276 & -1.78923 \\
\hline $\mathrm{C}$ & -0.61541 & 2.98012 & -0.21277 \\
\hline $\mathrm{H}$ & 0.46066 & 3.12633 & -0.28982 \\
\hline $\mathrm{H}$ & -0.90557 & 2.97408 & 0.83563 \\
\hline $\mathrm{N}$ & -0.89468 & 1.59657 & -0.74974 \\
\hline $\mathrm{C}$ & -1.92567 & 5.13171 & -0.12126 \\
\hline $\mathrm{H}$ & -2.53471 & 4.83034 & 0.73151 \\
\hline $\mathrm{C}$ & -1.65584 & 6.42493 & -0.30519 \\
\hline $\mathrm{H}$ & -2.03809 & 7.18959 & 0.36543 \\
\hline $\mathrm{H}$ & -1.04112 & 6.76975 & -1.13466 \\
\hline $\mathrm{C}$ & -2.42504 & 0.44036 & 0.97808 \\
\hline $\mathrm{C}$ & -3.87525 & 0.00407 & 1.14996 \\
\hline $\mathrm{C}$ & -4.37941 & -1.18391 & 0.51659 \\
\hline $\mathrm{C}$ & -4.76057 & 0.76628 & 1.88459 \\
\hline
\end{tabular}




\begin{tabular}{|c|c|c|c|}
\hline $\mathrm{C}$ & -5.78351 & -1.45694 & 0.66668 \\
\hline $\mathrm{C}$ & -3.58108 & -2.08401 & -0.23127 \\
\hline $\mathrm{C}$ & -6.12151 & 0.38499 & 1.98329 \\
\hline $\mathrm{H}$ & -4.42530 & 1.67304 & 2.37736 \\
\hline $\mathrm{C}$ & -6.32740 & -2.60265 & 0.03687 \\
\hline $\mathrm{C}$ & -4.15158 & -3.19558 & -0.83443 \\
\hline $\mathrm{H}$ & -2.50389 & -1.97014 & -0.30957 \\
\hline $\mathrm{H}$ & -6.80772 & 0.99811 & 2.56777 \\
\hline $\mathrm{C}$ & -5.54032 & -3.45500 & -0.70464 \\
\hline $\mathrm{H}$ & -7.38987 & -2.78511 & 0.16400 \\
\hline $\mathrm{H}$ & -5.98641 & -4.32449 & -1.17387 \\
\hline $\mathrm{N}$ & -6.63366 & -0.67286 & 1.39420 \\
\hline $\mathrm{O}$ & -3.30108 & -3.99552 & -1.53888 \\
\hline $\mathrm{C}$ & -3.80111 & -5.18914 & -2.11573 \\
\hline $\mathrm{H}$ & -4.56340 & -4.98620 & -2.88061 \\
\hline $\mathrm{H}$ & -2.94317 & -5.67291 & -2.58641 \\
\hline $\mathrm{H}$ & -4.22280 & -5.86246 & -1.35749 \\
\hline $\mathrm{N}$ & -2.00214 & 1.34787 & 2.05982 \\
\hline $\mathrm{H}$ & -2.37099 & 0.99024 & 2.93809 \\
\hline $\mathrm{H}$ & -0.98653 & 1.28319 & 2.14135 \\
\hline $\mathrm{H}$ & -1.79641 & -0.45725 & 0.94393 \\
\hline $\mathrm{H}$ & -0.20795 & 0.90286 & -0.38057 \\
\hline $\mathrm{O}$ & -0.34907 & -2.44761 & 0.79246 \\
\hline
\end{tabular}




$\begin{array}{llll}\mathrm{N} & 0.46564 & -1.73717 & 0.14719 \\ \mathrm{O} & 0.02743 & -0.87874 & -0.70732 \\ \mathrm{C} & 1.80245 & -1.90631 & 0.32964 \\ \mathrm{H} & 2.04775 & -2.71367 & 1.00320 \\ \mathrm{C} & 2.74191 & -1.05204 & -0.32742 \\ \mathrm{H} & 2.23314 & -0.45417 & -1.07745 \\ \mathrm{C} & 4.03245 & -1.62347 & -0.81234 \\ \mathrm{C} & 4.68729 & -2.69087 & -0.17580 \\ \mathrm{C} & 4.60828 & -1.09694 & -1.98173 \\ \mathrm{C} & 5.87627 & -3.20829 & -0.68742 \\ \mathrm{H} & 4.27081 & -3.12421 & 0.72778 \\ \mathrm{C} & 5.79325 & -1.61796 & -2.49979 \\ \mathrm{H} & 4.10599 & -0.28290 & -2.49856 \\ \mathrm{C} & 6.43416 & -2.67556 & -1.85170 \\ \mathrm{H} & 6.36733 & -4.02996 & -0.17364 \\ \mathrm{H} & 3.35234 & -0.84117 & 3.36166 \\ \mathrm{H} & 6.21293 & -1.20155 & -3.41189 \\ \mathrm{H} & 7.35903 & -3.08289 & -2.25101 \\ \mathrm{O} & 1.03402 & 1.07476 & 1.38772 \\ \mathrm{C} & 2.16288 & 0.70921 & 1.71134 \\ \mathrm{H} & 2.43768 & 0.37428 & 3.17497 \\ \mathrm{H} & 3.29701 & 0.55065 & 0.75966 \\ \mathrm{H} & 2.90776 & 1.26133 & 3.62762\end{array}$




$\begin{array}{llll}\mathrm{C} & 4.65119 & 0.31004 & 1.44030 \\ \mathrm{C} & 4.65022 & -0.70487 & 2.57863 \\ \mathrm{H} & 3.59325 & -1.01665 & 4.41510 \\ \mathrm{H} & 5.41338 & 0.00974 & 0.72053 \\ \mathrm{O} & 5.64275 & -1.34533 & 2.86605 \\ \mathrm{H} & 2.84130 & -1.74407 & 3.00283 \\ \mathrm{H} & 1.47391 & 0.23494 & 3.67190 \\ \mathrm{H} & 5.01897 & 1.25216 & 1.87992 \\ \mathrm{C} & 3.32801 & 1.58175 & -0.31536 \\ \mathrm{O} & 2.37937 & 1.97098 & -0.98388 \\ \mathrm{O} & 4.58462 & 2.05006 & -0.53661 \\ \mathrm{C} & 4.71272 & 3.00747 & -1.59458 \\ \mathrm{H} & 4.12273 & 3.90314 & -1.38026 \\ \mathrm{H} & 5.77467 & 3.25179 & -1.63984 \\ \mathrm{H} & 4.37668 & 2.58350 & -2.54516\end{array}$

TSII-6 (R,S)

$\begin{array}{llll}\mathrm{C} & -2.15852 & 1.16578 & -0.54819 \\ \mathrm{C} & -2.33084 & 3.17721 & -2.05308 \\ \mathrm{C} & -3.09442 & 2.28336 & -1.05019 \\ \mathrm{H} & -2.20569 & 0.30430 & -1.21444 \\ \mathrm{H} & -3.96634 & 1.82618 & -1.52725 \\ \mathrm{H} & -3.46595 & 2.87818 & -0.21008\end{array}$




\begin{tabular}{|c|c|c|c|}
\hline $\mathrm{C}$ & -0.39761 & 1.57957 & -2.21597 \\
\hline $\mathrm{H}$ & 0.57879 & 2.04845 & -2.34137 \\
\hline $\mathrm{H}$ & -0.31265 & 0.51182 & -2.42338 \\
\hline $\mathrm{C}$ & -1.52265 & 2.27881 & -3.01412 \\
\hline $\mathrm{H}$ & -1.08563 & 2.87605 & -3.82188 \\
\hline $\mathrm{H}$ & -2.18214 & 1.53912 & -3.48178 \\
\hline $\mathrm{H}$ & -3.02863 & 3.80874 & -2.61151 \\
\hline $\mathrm{C}$ & -1.31355 & 4.06514 & -1.28313 \\
\hline $\mathrm{H}$ & -0.60026 & 4.46985 & -2.01298 \\
\hline $\mathrm{C}$ & -0.57015 & 3.12760 & -0.29856 \\
\hline $\mathrm{H}$ & 0.50289 & 3.31418 & -0.25604 \\
\hline $\mathrm{H}$ & -0.99160 & 3.18803 & 0.70408 \\
\hline $\mathrm{N}$ & -0.72966 & 1.68916 & -0.74498 \\
\hline $\mathrm{C}$ & -1.93616 & 5.22856 & -0.55654 \\
\hline $\mathrm{H}$ & -2.64869 & 4.98087 & 0.23091 \\
\hline $\mathrm{C}$ & -1.66640 & 6.50964 & -0.81153 \\
\hline $\mathrm{H}$ & -2.14754 & 7.31489 & -0.26328 \\
\hline $\mathrm{H}$ & -0.95324 & 6.80346 & -1.57948 \\
\hline $\mathrm{C}$ & -2.42555 & 0.59892 & 0.87665 \\
\hline $\mathrm{C}$ & -3.89120 & 0.17989 & 0.96949 \\
\hline $\mathrm{C}$ & -4.36064 & -1.08922 & 0.48263 \\
\hline $\mathrm{C}$ & -4.81891 & 1.03543 & 1.53077 \\
\hline $\mathrm{C}$ & -5.76929 & -1.35650 & 0.61938 \\
\hline
\end{tabular}




$\begin{array}{llll}\mathrm{C} & -3.53012 & -2.07590 & -0.10226 \\ \mathrm{C} & -6.18258 & 0.66356 & 1.61247 \\ \mathrm{H} & -4.51066 & 2.00324 & 1.91241 \\ \mathrm{C} & -6.27852 & -2.59480 & 0.15921 \\ \mathrm{C} & -4.06865 & -3.27771 & -0.54097 \\ \mathrm{H} & -2.46038 & -1.93512 & -0.23419 \\ \mathrm{H} & -6.89992 & 1.35318 & 2.05772 \\ \mathrm{C} & -5.45667 & -3.54057 & -0.41068 \\ \mathrm{H} & -7.34346 & -2.76984 & 0.27536 \\ \mathrm{H} & -5.87640 & -4.48009 & -0.75192 \\ \mathrm{~N} & -6.65797 & -0.48168 & 1.17655 \\ \mathrm{O} & -3.18782 & -4.16131 & -1.08900 \\ \mathrm{C} & -3.66417 & -5.41548 & -1.54305 \\ \mathrm{H} & -4.40077 & -5.30560 & -2.35082 \\ \mathrm{H} & -1.70199 & -1.30763 & -0.88657 \\ \mathrm{H} & -2.78908 & -5.94289 & -1.92727 \\ \mathrm{H} & -4.10915 & -6.00308 & -0.72849 \\ \mathrm{~N} & -2.09453 & 1.58043 & 1.92711 \\ \mathrm{H} & -2.58004 & 1.31579 & 2.78071 \\ \mathrm{H} & -1.09948 & 1.48257 & 2.12680 \\ \mathrm{H} & -1.79745 & -0.29714 & 0.95989 \\ \mathrm{H} & -0.03993 & 1.14158 & -0.19846 \\ \mathrm{H} & -1.40221 & -0.02605\end{array}$




$\begin{array}{llll}\mathrm{O} & 0.77996 & 0.83359 & 1.49883 \\ \mathrm{C} & 1.79390 & 0.21612 & 1.81746 \\ \mathrm{C} & 3.09039 & 0.18480 & 1.07338 \\ \mathrm{C} & 4.28917 & -0.12293 & 1.99557 \\ \mathrm{H} & 4.07716 & 0.26320 & 3.00312 \\ \mathrm{C} & 3.23696 & 1.27975 & 0.07730 \\ \mathrm{O} & 2.29551 & 1.65034 & -0.62869 \\ \mathrm{C} & 5.54452 & 1.89579 & 1.04992 \\ \mathrm{C} & 5.62932 & 0.49271 & 1.61838 \\ \mathrm{C} & 4.61329 & 1.89789 & -0.17030 \\ \mathrm{O} & 6.68168 & -0.08211 & 1.81519 \\ \mathrm{O} & 1.85213 & -0.52178 & 2.94025 \\ \mathrm{C} & 0.61441 & -0.72593 & 3.63925 \\ \mathrm{H} & 0.22426 & 0.22290 & 4.01939 \\ \mathrm{H} & 5.08500 & 1.33027 & -0.98338 \\ \mathrm{H} & -0.10615 & -1.19519 & 2.96677 \\ \mathrm{H} & 0.86055 & -1.38871 & 4.46960 \\ \mathrm{H} & 4.43574 & -1.19822 & 2.12501 \\ \mathrm{H} & 6.54850 & 2.24777 & 0.79778 \\ \mathrm{H} & 5.13581 & 2.55866 & 1.82722 \\ \mathrm{H} & 4.44607 & 2.91073 & -0.55044 \\ \mathrm{H} & & -1.67233 & -0.71377 \\ \mathrm{H} & & -2.61499 & -0.17556\end{array}$




$\begin{array}{llll}\mathrm{C} & 4.49451 & -1.05191 & -1.92729 \\ \mathrm{C} & 6.23683 & -2.92043 & -0.81512 \\ \mathrm{H} & 4.77485 & -3.12612 & 0.74757 \\ \mathrm{C} & 5.69412 & -1.35724 & -2.56864 \\ \mathrm{H} & 3.82182 & -0.32509 & -2.37359 \\ \mathrm{C} & 6.57278 & -2.29137 & -2.01342 \\ \mathrm{H} & 6.90859 & -3.65165 & -0.37431 \\ \mathrm{H} & 5.94164 & -0.86721 & -3.50684 \\ \mathrm{H} & 7.50813 & -2.52752 & -2.51326 \\ \mathrm{H} & 2.66930 & -2.06096 & 0.82455 \\ \mathrm{H} & 1.75381 & -1.01120 & -1.92133 \\ \mathrm{~N} & 0.44508 & -1.57436 & -0.44401 \\ \mathrm{O} & 0.23762 & -1.99209 & 0.73356 \\ \mathrm{O} & -0.53106 & -1.39706 & -1.25201\end{array}$

TSII-7 (S,S)
$\begin{array}{llll}\text { C } & -2.15852 & 1.16578 & -0.54819\end{array}$
$\begin{array}{llll}\text { C } & -2.33084 & 3.17721 & -2.05308\end{array}$
$\begin{array}{llll}\text { C } & -3.09442 & 2.28336 & -1.05019\end{array}$
$\begin{array}{llll}\mathrm{H} & -2.20569 & 0.30430 & -1.21444\end{array}$
$\begin{array}{llll}\mathrm{H} & -3.96634 & 1.82618 & -1.52725\end{array}$
$\begin{array}{llll}\mathrm{H} & -3.46595 & 2.87818 & -0.21008\end{array}$
$\begin{array}{llll}\text { C } & -0.39761 & 1.57957 & -2.21597\end{array}$ 


\begin{tabular}{|c|c|c|c|}
\hline $\mathrm{H}$ & 0.57879 & 2.04845 & -2.34137 \\
\hline $\mathrm{H}$ & -0.31265 & 0.51182 & -2.42338 \\
\hline $\mathrm{C}$ & -1.52265 & 2.27881 & -3.01412 \\
\hline $\mathrm{H}$ & -1.08563 & 2.87605 & -3.82188 \\
\hline $\mathrm{H}$ & -2.18214 & 1.53912 & -3.48178 \\
\hline $\mathrm{H}$ & -3.02863 & 3.80874 & -2.61151 \\
\hline $\mathrm{C}$ & -1.31355 & 4.06514 & -1.28313 \\
\hline $\mathrm{H}$ & -0.60026 & 4.46985 & -2.01298 \\
\hline $\mathrm{C}$ & -0.57015 & 3.12760 & -0.29856 \\
\hline $\mathrm{H}$ & 0.50289 & 3.31418 & -0.25604 \\
\hline $\mathrm{H}$ & -0.99160 & 3.18803 & 0.70408 \\
\hline $\mathrm{N}$ & -0.72966 & 1.68916 & -0.74498 \\
\hline $\mathrm{C}$ & -1.93616 & 5.22856 & -0.55654 \\
\hline $\mathrm{H}$ & -2.64869 & 4.98087 & 0.23091 \\
\hline $\mathrm{C}$ & -1.66640 & 6.50964 & -0.81153 \\
\hline $\mathrm{H}$ & -2.14754 & 7.31489 & -0.26328 \\
\hline $\mathrm{H}$ & -0.95324 & 6.80346 & -1.57948 \\
\hline $\mathrm{C}$ & -2.42555 & 0.59892 & 0.87665 \\
\hline $\mathrm{C}$ & -3.89120 & 0.17989 & 0.96949 \\
\hline $\mathrm{C}$ & -4.36064 & -1.08922 & 0.48263 \\
\hline $\mathrm{C}$ & -4.81891 & 1.03543 & 1.53077 \\
\hline $\mathrm{C}$ & -5.76929 & -1.35650 & 0.61938 \\
\hline $\mathrm{C}$ & -3.53012 & -2.07590 & -0.10226 \\
\hline
\end{tabular}




\begin{tabular}{|c|c|c|c|}
\hline $\mathrm{C}$ & -6.18258 & 0.66356 & 1.61247 \\
\hline $\mathrm{H}$ & -4.51066 & 2.00324 & 1.91241 \\
\hline $\mathrm{C}$ & -6.27852 & -2.59480 & 0.15921 \\
\hline $\mathrm{C}$ & -4.06865 & -3.27771 & -0.54097 \\
\hline $\mathrm{H}$ & -2.46038 & -1.93512 & -0.23419 \\
\hline $\mathrm{H}$ & -6.89992 & 1.35318 & 2.05772 \\
\hline $\mathrm{C}$ & -5.45667 & -3.54057 & -0.41068 \\
\hline $\mathrm{H}$ & -7.34346 & -2.76984 & 0.27536 \\
\hline $\mathrm{H}$ & -5.87640 & -4.48009 & -0.75192 \\
\hline $\mathrm{N}$ & -6.65797 & -0.48168 & 1.17655 \\
\hline $\mathrm{O}$ & -3.18782 & -4.16131 & -1.08900 \\
\hline $\mathrm{C}$ & -3.66417 & -5.41548 & -1.54305 \\
\hline $\mathrm{H}$ & -4.40077 & -5.30560 & -2.35082 \\
\hline $\mathrm{H}$ & -2.78908 & -5.94289 & -1.92727 \\
\hline $\mathrm{H}$ & -4.10915 & -6.00308 & -0.72849 \\
\hline $\mathrm{N}$ & -2.09453 & 1.58043 & 1.92711 \\
\hline $\mathrm{H}$ & -2.58004 & 1.31579 & 2.78071 \\
\hline $\mathrm{H}$ & -1.09948 & 1.48257 & 2.12680 \\
\hline $\mathrm{H}$ & -1.79745 & -0.29714 & 0.95989 \\
\hline $\mathrm{H}$ & -0.03993 & 1.14158 & -0.19846 \\
\hline $\mathrm{C}$ & 1.70199 & -1.30763 & -0.88657 \\
\hline $\mathrm{C}$ & 2.83906 & -1.40221 & -0.02605 \\
\hline $\mathrm{O}$ & 0.77996 & 0.83359 & 1.49883 \\
\hline
\end{tabular}




$\begin{array}{llll}\mathrm{C} & 1.79390 & 0.21612 & 1.81746 \\ \mathrm{C} & 3.09039 & 0.18480 & 1.07338 \\ \mathrm{C} & 4.28917 & -0.12293 & 1.99557 \\ \mathrm{H} & 4.07716 & 0.26320 & 3.00312 \\ \mathrm{C} & 3.23696 & 1.27975 & 0.07730 \\ \mathrm{O} & 2.29551 & 1.65034 & -0.62869 \\ \mathrm{C} & 5.54452 & 1.89579 & 1.04992 \\ \mathrm{C} & 5.62932 & 0.49271 & 1.61838 \\ \mathrm{C} & 4.61329 & 1.89789 & -0.17030 \\ \mathrm{O} & 6.68168 & -0.08211 & 1.81519 \\ \mathrm{O} & 1.85213 & -0.52178 & 2.94025 \\ \mathrm{C} & 0.61441 & -0.72593 & 3.63925 \\ \mathrm{H} & 0.22426 & 0.22290 & 4.01939 \\ \mathrm{H} & -0.10615 & -1.19519 & 2.96677 \\ \mathrm{H} & 5.03450 & -2.61499 & -0.17556 \\ \mathrm{H} & 0.86055 & -1.38871 & 4.46960 \\ \mathrm{H} & 4.43574 & -1.19822 & 2.12501 \\ \mathrm{H} & 6.54850 & 2.24777 & 0.79778 \\ \mathrm{H} & 5.13581 & 2.55866 & 1.82722 \\ \mathrm{H} & 4.44607 & 2.91073 & -0.55044 \\ \mathrm{H} & 5.08500 & 1.33027 & -0.98338 \\ \mathrm{H} & 4.14479 & -1.67233 & -0.71377 \\ \mathrm{H} & & -1.05191 & -1.92729\end{array}$




$\begin{array}{llll}\mathrm{C} & 6.23683 & -2.92043 & -0.81512 \\ \mathrm{H} & 4.77485 & -3.12612 & 0.74757 \\ \mathrm{C} & 5.69412 & -1.35724 & -2.56864 \\ \mathrm{H} & 3.82182 & -0.32509 & -2.37359 \\ \mathrm{C} & 6.57278 & -2.29137 & -2.01342 \\ \mathrm{H} & 6.90859 & -3.65165 & -0.37431 \\ \mathrm{H} & 5.94164 & -0.86721 & -3.50684 \\ \mathrm{H} & 7.50813 & -2.52752 & -2.51326 \\ \mathrm{H} & 2.66930 & -2.06096 & 0.82455 \\ \mathrm{H} & 1.75381 & -1.01120 & -1.92133 \\ \mathrm{~N} & 0.44508 & -1.57436 & -0.44401 \\ \mathrm{O} & 0.23762 & -1.99209 & 0.73356 \\ \mathrm{O} & -0.53106 & -1.39706 & -1.25201\end{array}$

TSII-8 (S,R)

$\begin{array}{llll}\mathrm{C} & -2.13791 & 1.23990 & -0.40293 \\ \mathrm{C} & -2.27730 & 3.43133 & -1.63483 \\ \mathrm{C} & -3.06046 & 2.42157 & -0.76493 \\ \mathrm{H} & -2.18932 & 0.47293 & -1.17607 \\ \mathrm{H} & -3.93032 & 2.03911 & -1.30715 \\ \mathrm{H} & -3.43746 & 2.90654 & 0.14077 \\ \mathrm{C} & -0.35736 & 1.84542 & -1.98430 \\ \mathrm{H} & 0.62876 & 2.30990 & -2.04014\end{array}$




\begin{tabular}{|c|c|c|c|}
\hline $\mathrm{H}$ & -0.28853 & 0.81315 & -2.32965 \\
\hline $\mathrm{C}$ & -1.46290 & 2.66155 & -2.69741 \\
\hline $\mathrm{H}$ & -1.00805 & 3.35758 & -3.41045 \\
\hline $\mathrm{H}$ & -2.12151 & 1.99875 & -3.26990 \\
\hline $\mathrm{H}$ & -2.96303 & 4.13646 & -2.11452 \\
\hline $\mathrm{C}$ & -1.26109 & 4.20204 & -0.74535 \\
\hline $\mathrm{H}$ & -0.53802 & 4.69318 & -1.40956 \\
\hline $\mathrm{C}$ & -0.53519 & 3.13619 & 0.11228 \\
\hline $\mathrm{H}$ & 0.53997 & 3.30186 & 0.18985 \\
\hline $\mathrm{H}$ & -0.96613 & 3.06768 & 1.11034 \\
\hline $\mathrm{N}$ & -0.70512 & 1.77048 & -0.51839 \\
\hline $\mathrm{C}$ & -1.88383 & 5.26531 & 0.12115 \\
\hline $\mathrm{H}$ & -2.59924 & 4.92150 & 0.86920 \\
\hline $\mathrm{C}$ & -1.61241 & 6.56803 & 0.03069 \\
\hline $\mathrm{H}$ & -2.09472 & 7.29809 & 0.67477 \\
\hline $\mathrm{H}$ & -0.89670 & 6.95598 & -0.69174 \\
\hline $\mathrm{C}$ & -2.41682 & 0.49317 & 0.93492 \\
\hline $\mathrm{C}$ & -3.88566 & 0.07904 & 0.96534 \\
\hline $\mathrm{C}$ & -4.36074 & -1.11313 & 0.31724 \\
\hline $\mathrm{C}$ & -4.81064 & 0.86393 & 1.62548 \\
\hline $\mathrm{C}$ & -5.77272 & -1.38162 & 0.40541 \\
\hline $\mathrm{C}$ & -3.53166 & -2.02660 & -0.37873 \\
\hline $\mathrm{C}$ & -6.17826 & 0.49817 & 1.64654 \\
\hline
\end{tabular}




$\begin{array}{llll}\mathrm{H} & -4.49638 & 1.77045 & 2.13231 \\ \mathrm{C} & -6.28728 & -2.54679 & -0.21283 \\ \mathrm{C} & -4.07519 & -3.15855 & -0.97018 \\ \mathrm{H} & -2.45882 & -1.88136 & -0.47870 \\ \mathrm{H} & -6.89406 & 1.13157 & 2.17072 \\ \mathrm{C} & -5.46695 & -3.42164 & -0.88823 \\ \mathrm{H} & -7.35482 & -2.72492 & -0.13014 \\ \mathrm{H} & -5.89051 & -4.30697 & -1.34856 \\ \mathrm{~N} & -6.65940 & -0.57615 & 1.06145 \\ \mathrm{O} & -3.19597 & -3.97558 & -1.61579 \\ \mathrm{C} & -3.67701 & -5.16374 & -2.21891 \\ \mathrm{H} & -4.40372 & -4.95306 & -3.01554 \\ \mathrm{H} & -2.80180 & -5.65031 & -2.65341 \\ \mathrm{H} & -4.13552 & -5.83841 & -1.48327 \\ \mathrm{H} & -0.75678 & 0.64129 & 1.47660 \\ \mathrm{H} & -2.07891 & 1.32180 & 2.10644 \\ \mathrm{H} & -2.56522 & 0.94670 & 2.91729 \\ \mathrm{H} & -1.08337 & 1.18390 & 2.28573 \\ \mathrm{H} & -1.79686 & -0.41197 & 0.90129 \\ \mathrm{H} & -0.03138 & 1.15025 & -0.02244 \\ \mathrm{H} & 1.76520 & -1.10735 & -1.10853 \\ \mathrm{H} & 2.94204 & -1.31757 & -0.34707 \\ \mathrm{H} & -0.04179 & 1.84909\end{array}$




$\begin{array}{llll}\mathrm{C} & 1.59407 & -0.87351 & 3.11946 \\ \mathrm{C} & 3.04758 & -0.01553 & 1.19357 \\ \mathrm{C} & 2.42657 & -2.15844 & 3.10028 \\ \mathrm{H} & 1.91172 & -0.23174 & 3.95745 \\ \mathrm{C} & 4.17690 & -0.55144 & 2.08074 \\ \mathrm{C} & 3.87289 & -1.91471 & 2.69475 \\ \mathrm{H} & 2.41620 & -2.66992 & 4.06896 \\ \mathrm{H} & 5.12076 & -0.61746 & 1.53855 \\ \mathrm{O} & 4.73857 & -2.75646 & 2.85054 \\ \mathrm{H} & 1.98910 & -2.85244 & 2.37129 \\ \mathrm{H} & 0.53397 & -1.09171 & 3.26635 \\ \mathrm{H} & 4.35743 & 0.13949 & 2.92128 \\ \mathrm{C} & 3.32865 & 1.24926 & 0.46706 \\ \mathrm{O} & 2.55734 & 1.84154 & -0.27666 \\ \mathrm{C} & 4.47867 & -0.28338 & -2.08781 \\ \mathrm{O} & 4.59113 & 1.69462 & 0.68975 \\ \mathrm{C} & 4.97138 & 2.87495 & -0.02684 \\ \mathrm{H} & 4.29825 & 3.70483 & 0.20596 \\ \mathrm{C} & 5.98624 & 3.10148 & 0.30233 \\ \mathrm{C} & 4.95476 & 2.69173 & -1.10466 \\ \mathrm{H} & 4.25413 & -1.21029 & -1.05373 \\ \mathrm{H} & 5.29698 & -2.08626 & -0.70846 \\ \mathrm{H} & -2.04591 & -1.37971\end{array}$




$\begin{array}{llll}\mathrm{H} & 5.15080 & -2.80268 & 0.09525 \\ \mathrm{C} & 5.70029 & -0.24375 & -2.75756 \\ \mathrm{H} & 3.69624 & 0.41694 & -2.36203 \\ \mathrm{C} & 6.72627 & -1.12615 & -2.40822 \\ \mathrm{H} & 7.30977 & -2.73661 & -1.09662 \\ \mathrm{H} & 5.85079 & 0.47533 & -3.55893 \\ \mathrm{H} & 7.67680 & -1.09566 & -2.93409 \\ \mathrm{~N} & 0.55605 & -1.51951 & -0.63586 \\ \mathrm{O} & 0.47584 & -2.15054 & 0.45764 \\ \mathrm{O} & -0.48956 & -1.24306 & -1.31442 \\ \mathrm{H} & 1.72944 & -0.57446 & -2.04428 \\ \mathrm{H} & 2.88036 & -2.18675 & 0.30080\end{array}$




\section{References}

1. (a) A. D. Becke, J. Chem. Phys., 1993, 98, 1372; (b) A. D. Becke, J. Chem. Phys., 1993, 98, 5648; (c) C. Lee, W. Yang, R. G. Parr, Phys. Rev. B, 1988, 37, 785.

2. R. Ditchfield, W. J. Hehre, J. A. Pople, J. Chem. Phys., 1971, 54, 724.

3. P. C. Hariharan, J. A. Pople, Theor. Chem. Acc., 1973, 28, 213.

4. M. J. Frisch, G. W. Trucks, H. B. Schlegel, G. E. Scuseria, M. A. Rob, J. R. Cheeseman, J. A. Montgomery Jr., T. Vreven, K. N. Kudin, J. C. Burant, J. M. Millam, S. S. Iyengar, J. Tomasi, V. Barone, B. Mennucci, M. Cossi, G. Scalmani, N. Rega, G. A. Petersson, H. Nakatsuji, M. Hada, M. Ehara, K. Toyota, R. Fukuda, J. Hasegawa, M. Ishida, T. Nakajima, Y. Honda, O. Kitao, H. Nakai, M. Klene, X. Li, J. E. Knox, H. P. Hratchian, J. B. Cross, V. Bakken, C. Adamo, J. Jaramillo, R. Gomperts, R. E. Stratmann, O. Yazyev, A. J. Austin, R. Cammi, C. Pomelli, J. W. Ochterski, P. Y. Ayala, K. Morokuma, G. A. Voth, P. Salvador, J. J. Dannenberg, V. G. Zakrzewski, S. Dapprich, A. D. Daniels, M. C. Strain, O. Farkas, D. K. Malick, A. D. Rabuck, K. Raghavachari, J. B. Foresman, J. V. Ortiz, Q. Cui, A. G. Baboul, S. Clifford, J. Cioslowski, B. B. Stefanov, G. Liu, A. Liashenko, P. Piskorz, I. Komaromi, R. L. Martin, D. J. Fox, T. Keith, M. A. Al-Laham, C. Y. Peng, A. Nanayakkara, M. Challacombe, P. M. W. Gill, B. Johnson, W. Chen, M. W. Wong, C. Gonzalez, and J. A. Pople, Gaussian 03, Revision E.01 (Gaussian, Inc., Wallingford, CT, 2003). 